Experimental investigation of ion cyclotron range of frequencies heating scenarios for ITER's half-field hydrogen phase performed in JET

This article has been downloaded from IOPscience. Please scroll down to see the full text article.

2012 Plasma Phys. Control. Fusion 54074008

(http://iopscience.iop.org/0741-3335/54/7/074008)

View the table of contents for this issue, or go to the journal homepage for more

Download details:

IP Address: 193.190.204.60

The article was downloaded on 11/07/2012 at 14:09

Please note that terms and conditions apply. 


\title{
Experimental investigation of ion cyclotron range of frequencies heating scenarios for ITER's half-field hydrogen phase performed in JET
}

\author{
E Lerche $^{1}$, D Van Eester ${ }^{1}$, T J Johnson ${ }^{2}$, T Hellsten ${ }^{2}$, J Ongena ${ }^{1}$, \\ M-L Mayoral ${ }^{3}$, D Frigione ${ }^{4}, \mathrm{C} \mathrm{Sozzi}^{4}$, G Calabro ${ }^{4}$, M Lennholm $^{3}$,

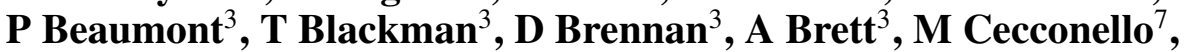 \\ I Coffey $^{3}$, A Coyne $^{3}$, K Crombe $^{1}$, A Czarnecka $^{5}$, R Felton $^{3}$, C Giroud $^{3}$, \\ G Gorini $^{6}$, C Hellesen ${ }^{7}$, P Jacquet ${ }^{3}$, V Kiptily ${ }^{3}$, S Knipe ${ }^{3}$, \\ A Krasilnikov ${ }^{8}, M_{\text {Maslov }}^{3}$, I Monakhov ${ }^{3}$, C Noble $^{3}$, M Nocente $^{6}$,

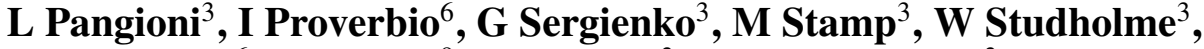 \\ M Tardocchi ${ }^{6}$, V Vdovin ${ }^{9}$, T Versloot ${ }^{3}$, I Voitsekhovitch ${ }^{3}$, \\ A Whitehurst $^{3}$, E Wooldridge ${ }^{3}$, V Zoita ${ }^{10}$ and JET EFDA \\ Contributors $^{11}$ \\ JET-EFDA Culham Science Centre, Abingdon, OX14 3DB, UK \\ ${ }^{1}$ LPP-ERM/KMS, Association Euratom-'Belgian State', TEC Partner, Brussels, Belgium \\ ${ }^{2}$ Fusion Plasma Physics, Association Euratom-VR, KTH, Stockholm, Sweden \\ ${ }^{3}$ Euratom-CCFE Fusion Association, Culham Science Centre, Abingdon, UK \\ ${ }^{4}$ Euratom-ENEA sulla Fusione, C. R. Frascati, Frascati, Italy \\ ${ }^{5}$ Institute of Plasma Physics and Laser Microfusion, Warsaw, Poland \\ ${ }^{6}$ Instituto di Fisica del Plasma, EURATOM-ENEA-CNR Association, Milan, Italy \\ ${ }^{7}$ Department of Physics and Astronomy, Uppsala University, Association EURATOM-VR, \\ Uppsala, Sweden \\ ${ }^{8}$ SRC RF Troitsk Institute for Innovating and Fusion Research, Troitsk, Russia \\ ${ }^{9}$ RNC Kurchatov Institute, Nuclear Fusion Institute, Moscow, Russia \\ ${ }^{10}$ Association EURATOM-MEdC, National Institute for Plasma Physics, Bucharest, Romania
}

Received 1 September 2011, in final form 30 January 2012

Published 20 June 2012

Online at stacks.iop.org/PPCF/54/074008

\footnotetext{
Abstract

Two ion cyclotron range of frequencies (ICRF) heating schemes proposed for the half-field operation phase of ITER in hydrogen plasmas - fundamental $\mathrm{H}$ majority and second harmonic ${ }^{3} \mathrm{He}$ ICRF heating - were recently investigated in JET. Although the same magnetic field and $\mathrm{RF}$ frequencies ( $f \approx 42 \mathrm{MHz}$ and $f \approx 52 \mathrm{MHz}$, respectively) were used, the density and particularly the plasma temperature were lower than those expected in the initial phase of ITER. Unlike for the well-performing $\mathrm{H}$ minority heating scheme to be used in ${ }^{4} \mathrm{He}$ plasmas, modest heating efficiencies $\left(\eta=P_{\text {absorbed }} / P_{\text {launched }}<40 \%\right)$ with dominant electron heating were found in both $\mathrm{H}$ plasma scenarios studied, and enhanced plasma-wall interaction manifested by high radiation losses and relatively large impurity content in the plasma was observed. This effect was stronger in the ${ }^{3} \mathrm{He}$ ICRF heating case than in the $\mathrm{H}$ majority heating experiments and it was verified that concentrations as high as $\sim 20 \%$ are necessary to observe significant ion heating in this case. The RF acceleration of the heated ions was modest in both cases, although a small fraction of the ${ }^{3} \mathrm{He}$ ions reached about $260 \mathrm{keV}$ in the second harmonic ${ }^{3} \mathrm{He}$ heating experiments when

${ }^{11}$ Romanelli F et al 2010 Proc. 23rd IAEA Fusion Energy Conf. (Daejeon, Korea, 2010) paper OV-1/3.
} 
$5 \mathrm{MW}$ of ICRF power was applied. Considerable RF acceleration of deuterium beam ions was also observed in some discharges of the ${ }^{3} \mathrm{He}$ heating experiments (where both the second and third harmonic ion cyclotron resonance layers of the $\mathrm{D}$ ions are inside the plasma) whilst it was practically absent in the majority hydrogen heating scenario. While hints of improved RF heating efficiency as a function of the plasma temperature and plasma dilution (with ${ }^{4} \mathrm{He}$ ) were confirmed in the $\mathrm{H}$ majority case, the ${ }^{3} \mathrm{He}$ concentration was the main handle on the heating efficiency in the second harmonic ${ }^{3} \mathrm{He}$ heating scenario.

(Some figures may appear in colour only in the online journal)

\section{Introduction}

\subsection{Motivation}

The non-active phase of ITER [1] will start with hydrogen plasmas at a reduced magnetic field. At the nominal halffield of $B_{0}=2.65 \mathrm{~T}$ and with the auxiliary power currently foreseen to be available in this phase, $16.5 \mathrm{MW}$ off-axis neutral beam injection (NBI), $15 \mathrm{MW}$ off-axis electron cyclotron resonance heating $(\mathrm{ECRH})$ and $10 \mathrm{MW}$ on-axis ion cyclotron resonance heating (ICRH) or radio-frequency (RF) heating, the discharges are expected to be in L-mode, and typical central densities of $n_{0} \approx 3 \times 10^{19} \mathrm{~m}^{-3}$ and central temperatures of approximately $T_{\mathrm{i}}=8 \mathrm{keV}$ and $T_{\mathrm{e}}=10 \mathrm{keV}$ have been estimated [2]. In these calculations, it was estimated that $10 \mathrm{MW}$ of ICRF power would be sufficient for raising the ion and electron temperatures from $T_{\mathrm{i}} \approx T_{\mathrm{e}}=5 \mathrm{keV}$ (calculated without ICRH) to $T_{\mathrm{i}}=8 \mathrm{keV} / T_{\mathrm{e}}=10 \mathrm{keV}$, if central ion cyclotron absorption with equal power sharing between electrons and ions is considered. This is a rather optimistic statement and is based on the assumption that all the power launched by the ICRF antenna is absorbed in the plasma. In $\mathrm{H}$ plasmas and for the designed frequency range of the ICRF heating system in ITER ( $f=40-55 \mathrm{MHz}$ ) [3], only fundamental ion cyclotron heating of $\mathrm{H}$ ions (at $f \approx 40 \mathrm{MHz}$ ) and second harmonic $(N=2)$ ion cyclotron heating of ${ }^{3} \mathrm{He}$ (at $f \approx 53 \mathrm{MHz}$ ) ions are feasible for central ion heating at the nominal half-field value of $B_{0}=2.65 \mathrm{~T}$. This is illustrated in figure 1, where the radial positions of the cold ion cyclotron layers of a few ion species are plotted as a function of the RF frequency for the ITER parameters at $B_{0}=2.65 \mathrm{~T}$. The continuous lines represent fundamental $(N=1)$ ion cyclotron resonances, the dashed lines represent the second harmonic $(N=2)$ resonances and the dotted lines stand for the third harmonic $(N=3)$ ion cyclotron resonances of the various species. Note that the impurities' resonances (with charge over mass somewhat lower than 1/2) are always located at the high-field side of the machine.

It is important to mention that none of the two ICRF scenarios cited above are high-performance heating schemes from the RF point of view: The fundamental $\mathrm{H}$ majority scenario suffers from the 'adverse' polarization of the RF fields close to the ion cyclotron layer of the majority $\mathrm{H}$ ions ('screening effect') whereas the second harmonic ${ }^{3} \mathrm{He}$ heating scheme relies on a relatively large fraction of 'minority' ions to become efficient. Preliminary numerical simulations of these ICRH scenarios for ITER's half-field phase in H plasmas indicate that relatively low single-pass RF power absorption

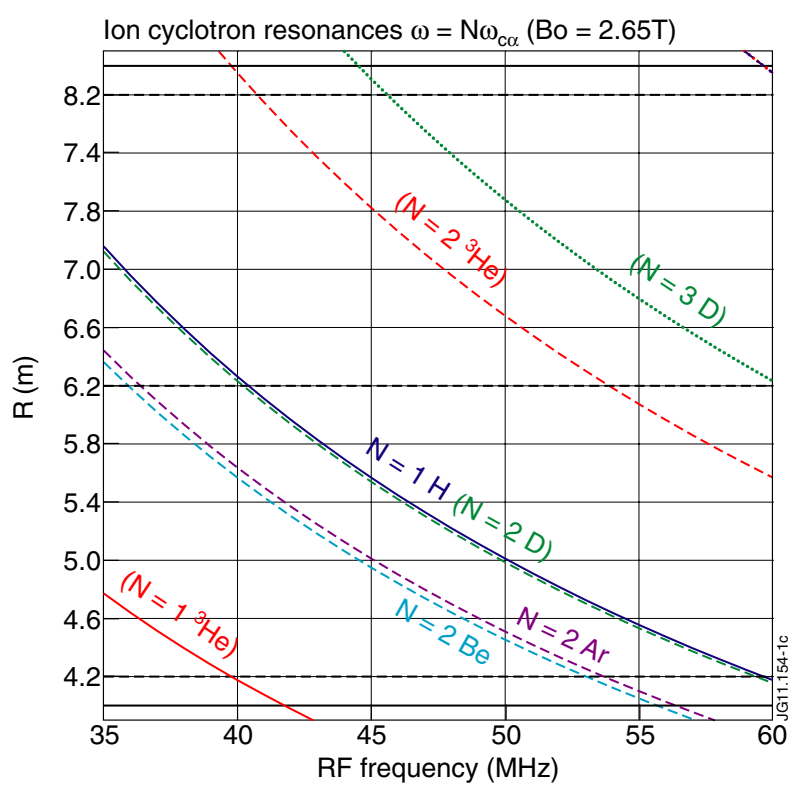

Figure 1. Fundamental (solid), second harmonic (dashed) and third harmonic (dotted) ion cyclotron resonance positions of several ion species as a function of the ICRF frequency for the ITER half-field scenario $\left(B_{0}=2.65 \mathrm{~T}\right)$. The operational frequency range of the ICRF system will be from $f=40-55 \mathrm{MHz}$.

$(<50 \%)$ with dominant fast-wave electron heating will take place, and that high heating efficiencies-as those typically observed for fundamental ICRF minority heating-will be unlikely. Given the fact that the initial ITER operation relies heavily on every MW of auxiliary heating power that can be injected into the plasma, experiments aiming at assessing the ICRF heating performance of the ITER half-field scenarios in $\mathrm{H}$ plasmas were recently performed in JET.

\subsection{Experimental set-up}

The ICRF parameters of the half-field phase of ITER were closely reproduced in the JET experiments [4]: The fundamental hydrogen heating scenario was studied at $f=$ $42.5 \mathrm{MHz}$ and the $N=2{ }^{3} \mathrm{He}$ ICRH experiments were done at $f=51.5 \mathrm{MHz}$, both using $B_{0}=2.65 \mathrm{~T}$. Under these conditions, the fundamental ion cyclotron resonance layer of the $\mathrm{H}$ ions is located around $R=2.85 \mathrm{~m}$ whereas the $N=2$ ion cyclotron resonance of the ${ }^{3} \mathrm{He}$ ions is located at approximately $R=3.15 \mathrm{~m}$, respectively $\left(R_{0}=2.98 \mathrm{~m}\right.$ being the centre of the plasma). This is illustrated in figure 2 , where the ion cyclotron layers of $\mathrm{H}, \mathrm{D}$ and ${ }^{3} \mathrm{He}$ for $f=42.5 \mathrm{MHz}(a)$ 

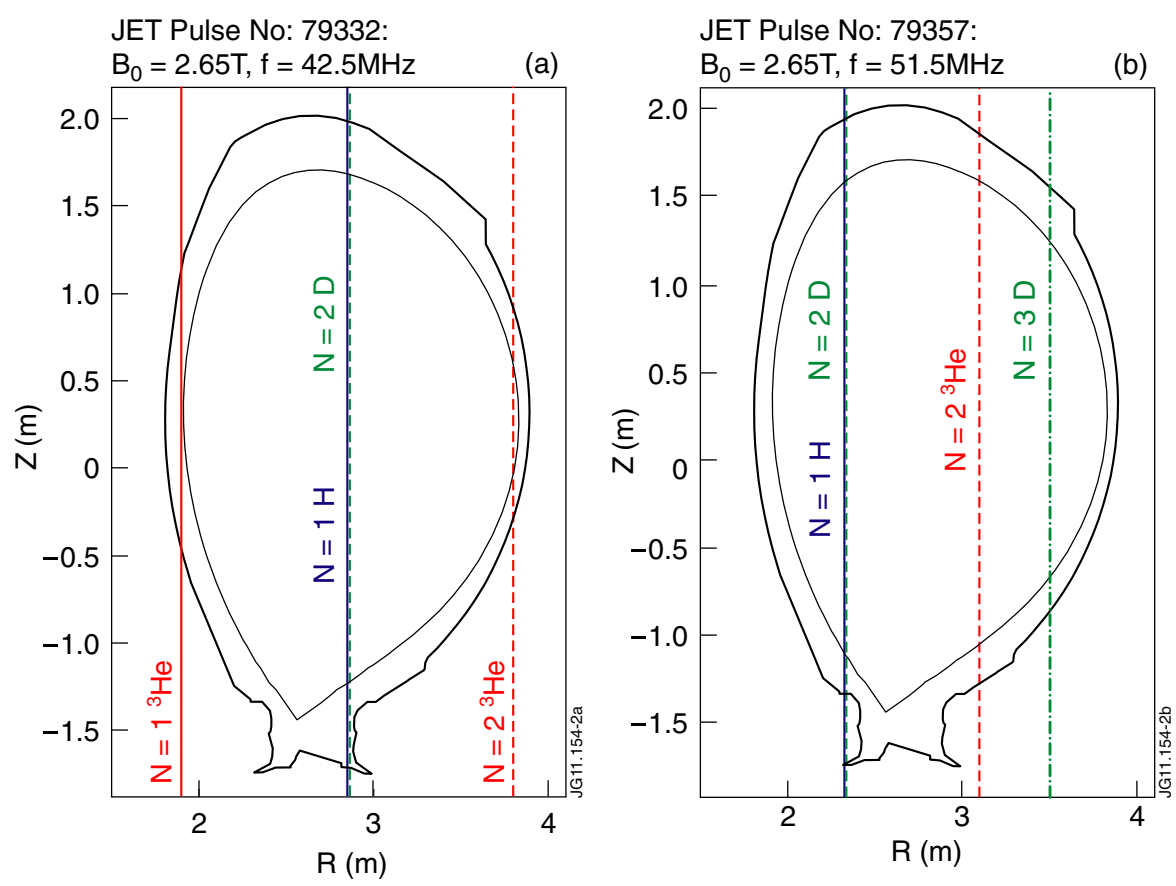

ITER:

(c)
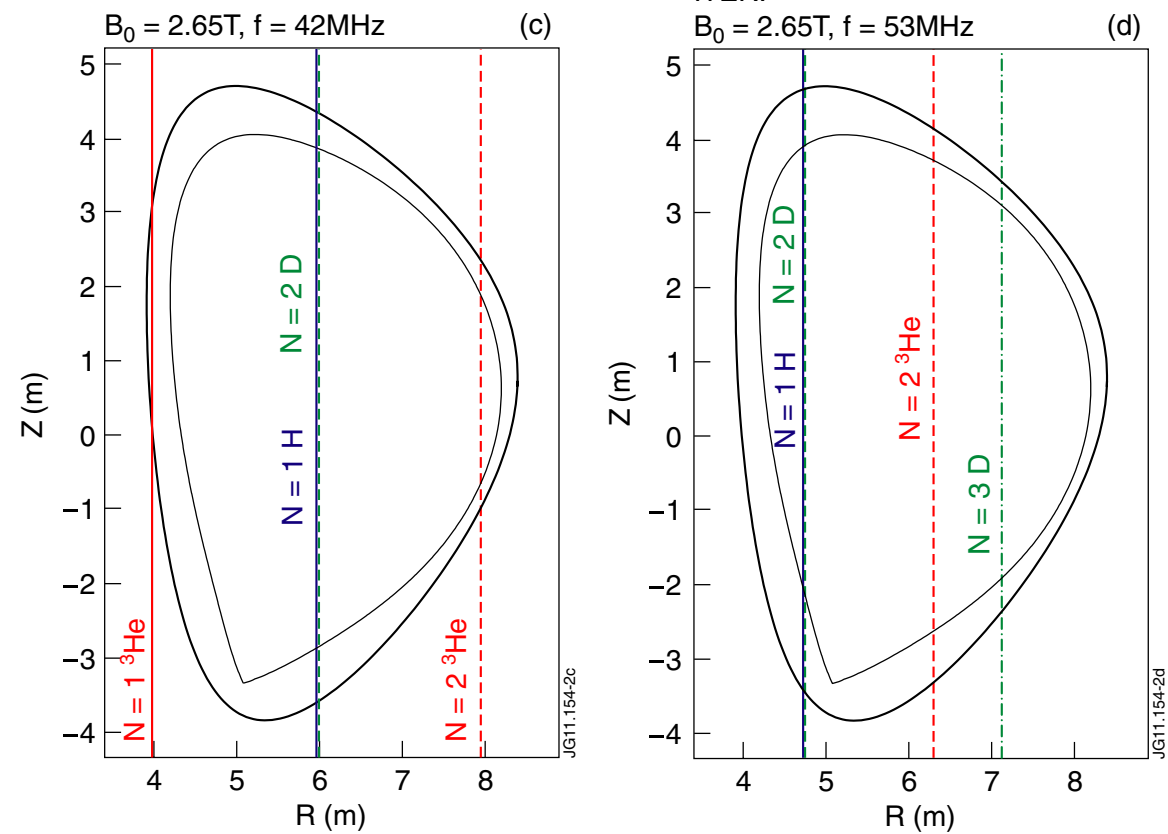

Figure 2. $(a),(b)$ Ion cyclotron resonance positions of $\mathrm{H}, \mathrm{D}$ and ${ }^{3} \mathrm{He}$ at $B_{0}=2.65 \mathrm{~T}$ for the fundamental $\mathrm{H}$ ICRH scheme at $f=42.5 \mathrm{MHz}$ $(a)$ and for the $N=2{ }^{3} \mathrm{He}$ ICRH scheme at $f=51.5 \mathrm{MHz}(b)$ in two JET discharges. $(c),(d)$ Equivalent resonance positions in ITER during the half-field operation phase with similar RF frequencies.

and for $f=51.5 \mathrm{MHz}(b)$ are represented in a poloidal crosssection of the JET tokamak. Figures $2(c)$ and $(d)$ represent the equivalent cyclotron resonance positions for ITER in its half-field operation phase at similar RF frequencies.

Note that at $f=42.5 \mathrm{MHz}$, the fundamental $(N=1)$ and second harmonic $(N=2)$ resonances of ${ }^{3} \mathrm{He}$ are located close to the plasma edge at the high-field side (HFS) and low-field side (LFS), respectively. At $f=51.5 \mathrm{MHz}$, the fundamental $\mathrm{H}$ (together with second harmonic $\mathrm{D}$ ) resonance is located at mid-radius to the HFS and the $N=3$ harmonic D resonance at mid-radius to the LFS. In practice, this means that the fundamental H ICRH discharges have to be done without
${ }^{3} \mathrm{He}$ to avoid resonant acceleration of the ${ }^{3} \mathrm{He}$ ions near the edge while in the $N=2{ }^{3} \mathrm{He}$ heating scenario, parasitic absorption of $\mathrm{H}$ and particularly of fast $\mathrm{D}$ beam ions can be expected.

The four A2 ICRF antennas [5] (composed of four straps each) were used in a dipole configuration $(0 \pi 0 \pi$, launching a symmetric toroidal spectrum with maximum power at $\left|n_{\phi}\right|=$ 27 ) in both experiments and up to $5.5 \mathrm{MW}$ of ICRF power was coupled to the plasma. Apart from the different ICRF parameters and the dilution of the $\mathrm{H}$ plasmas with ${ }^{3} \mathrm{He}$ in the $N=2{ }^{3} \mathrm{He}$ ICRH pulses, the plasmas were similar in the two experiments. Both were performed in L-mode and adopted a plasma geometry that favours the ICRF antenna coupling, with 

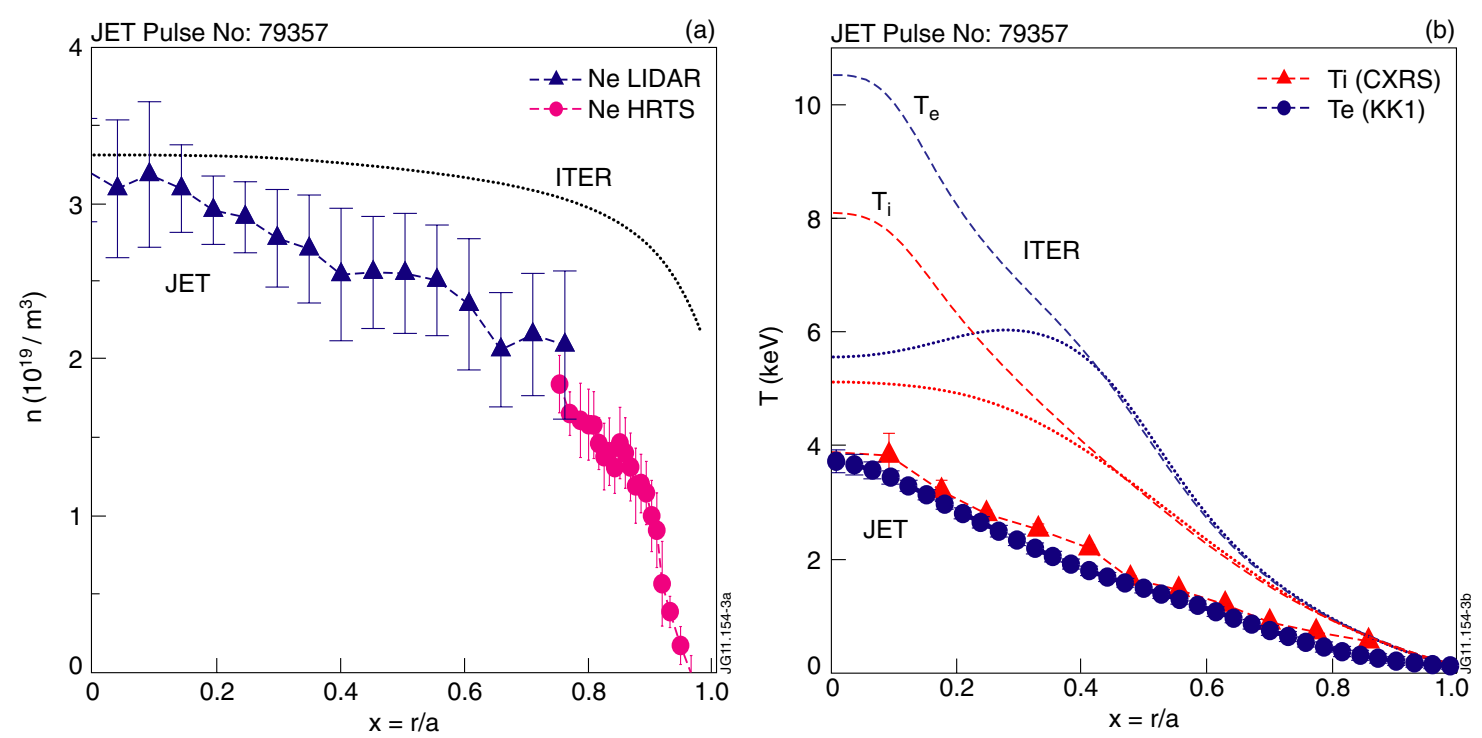

Figure 3. (a) Typical density profile of the JET experiments measured with Thomson scattering diagnostics (LIDAR—core, HRTS—edge) compared with the density expected in the half-field ITER phase (dotted line). (b) Electron and ion temperature profiles measured, respectively, with ECE radiometry and CXRS in JET. The dotted lines represent the temperature profiles expected in the initial phase of ITER without ICRH (31.5 MW: NBI + ECRH only) while the dashed lines correspond to the temperature profiles computed for the total auxiliary power foreseen to be available in this phase (41.5 MW: ICRH + NBI + ECRH).

antenna strap-separatrix distances varying between 9.5 and $11.0 \mathrm{~cm}$. Fast current ramp-up associated with NBI pre-heating was used to delay the appearance of sawteeth oscillations in most discharges.

Central densities of $n_{0}=3 \times 10^{19} \mathrm{~m}^{-3}$ and central temperatures ranging from $T_{\mathrm{e}}=2-4 \mathrm{keV}$, depending on the NBI power applied $\left(0<P_{\mathrm{NBI}}<8 \mathrm{MW}\right)$, were typical of the experiments. An example of the characteristic density profiles and temperature profiles of the JET discharges is shown, respectively, in figures $3(a)$ and $(b)$, together with the profiles calculated for the half-field ITER operation in $\mathrm{H}$ plasmas with $31.5 \mathrm{MW}$ (no ICRH-dotted lines) and 41.5 MW (dashed lines) of auxiliary power [2]. Note that although the central densities of the JET experiments are comparable to those expected in the initial phase of ITER, both electron and ion temperatures are well below the target ITER values (even without ICRF) leading to a completely different collisional regime than the one expected in ITER.

Since there were no hydrogen beams available for the experiments, deuterium NBI [6] was used both for diagnostic purposes (charge-exchange recombination spectroscopy (CXRS), motional Stark effect measurements) and to pre-heat the L-mode plasma. The selection of NBI PINIs (positive ion neutral injectors) was done to minimize the harmonic ion cyclotron absorption of the beam deuterons in the two scenarios, i.e. by using off-axis and low-energy ( $\sim 80 \mathrm{keV})$ PINIs. Each NBI PINI corresponds to $\sim 1.3 \mathrm{MW}$ of auxiliary NBI power with these settings. The fundamental $\mathrm{H}$ majority heating experiments were performed first and no ${ }^{3} \mathrm{He}$ was injected in the machine to avoid spurious ${ }^{3} \mathrm{He}$ absorption/acceleration near the plasma edge. Apart from the deuterium fuelling from NBI injection and wall recycling $(X[\mathrm{D}]<10 \%)$, there were traces of ${ }^{4} \mathrm{He}$ ions in the plasma $\left(X\left[{ }^{4} \mathrm{He}\right]<4 \%\right)$ reminiscent from previous ${ }^{4} \mathrm{He}$ plasmas performed a few days before the experiments reported here. In the second harmonic ${ }^{3} \mathrm{He}$ heating experiments, the injection of ${ }^{3} \mathrm{He}$ was controlled using a PID (proportional-integralderivative) feedback control on the gas valve actuation based on the real-time measurements of line emission intensities of several ion species by visible spectroscopy [7]. The ${ }^{3} \mathrm{He}$ concentration $X\left[{ }^{3} \mathrm{He}\right]$ was varied between $5 \%$ and $25 \%$ in these experiments. Although the spectroscopic measurements do not discriminate between ${ }^{3} \mathrm{He}$ and ${ }^{4} \mathrm{He}$, it was verified that the ${ }^{4} \mathrm{He}$ level had already decreased below $1 \%$ when the ${ }^{3} \mathrm{He}$ ICRH experiments started.

Apart from the standard data routinely available in JET, the main diagnostics used for the studies reported here were the ECE heterodyne radiometer (KK3) [8] and CXRS (KS5) [9] for electron and ion temperature measurements, respectively, and the horizontal (KR2) and vertical (KF1) neutral particle analysers (NPAs) [10,11] for the assessment of fast ion tails eventually created due to ICRF acceleration. The latter were complemented by measurements of fast ion losses performed with the scintillator probe KA3 [12]. Additionally, $\gamma$-ray spectrometry (KM6) [13] and the $2.5 \mathrm{MeV}$ neutron spectrometer TOFOR $[14,15]$ assisted in monitoring whether or not fast ions were present in the discharges. Finally, the study of the impurity release during ICRF operation was based on the analysis of the total radiation measured by the KB5 bolometer [16] and on both visible edge spectroscopy (KS3) [17] and VUV spectroscopy (KT2/KT7) [18] data.

In the next two sections, the experimental results of the fundamental H ICRH experiments and of the second harmonic ${ }^{3} \mathrm{He}$ heating experiments performed at JET will be presented, respectively. In section 4 , the overall performance of the two heating scenarios is discussed, including a comparison of the impurity generation due to ICRF in the two cases. The paper ends with a summary of the main achievements and 

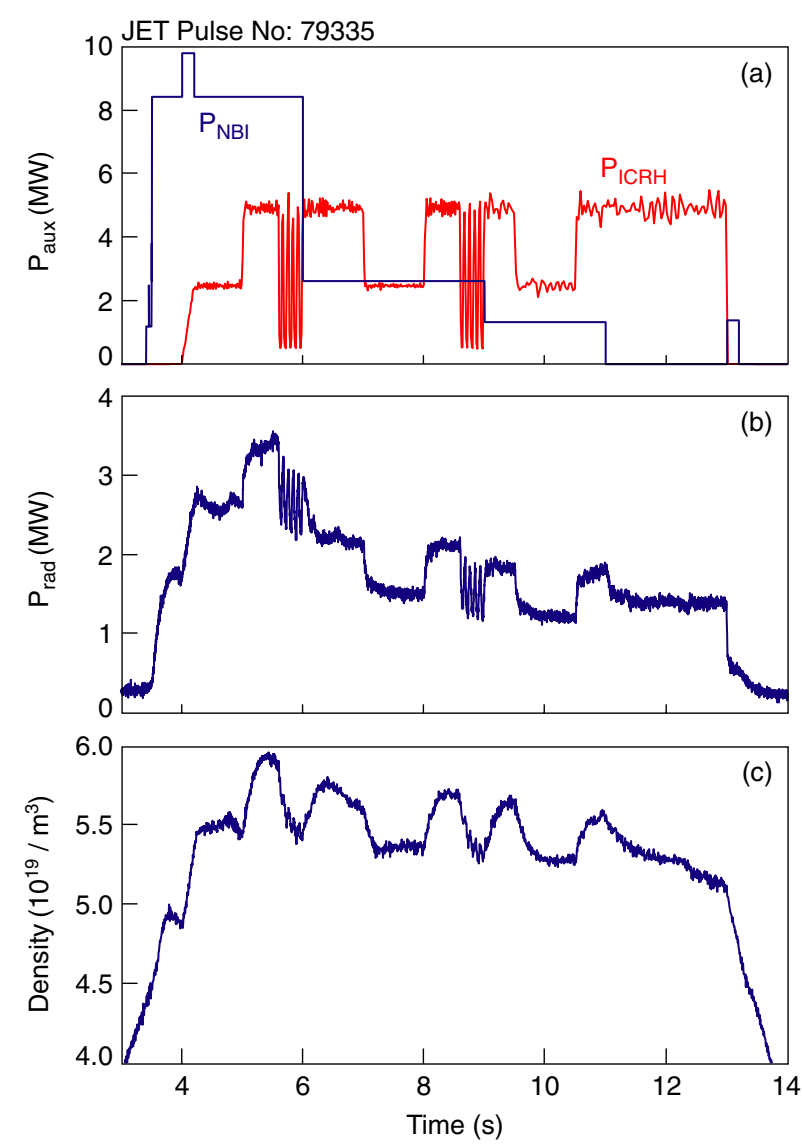
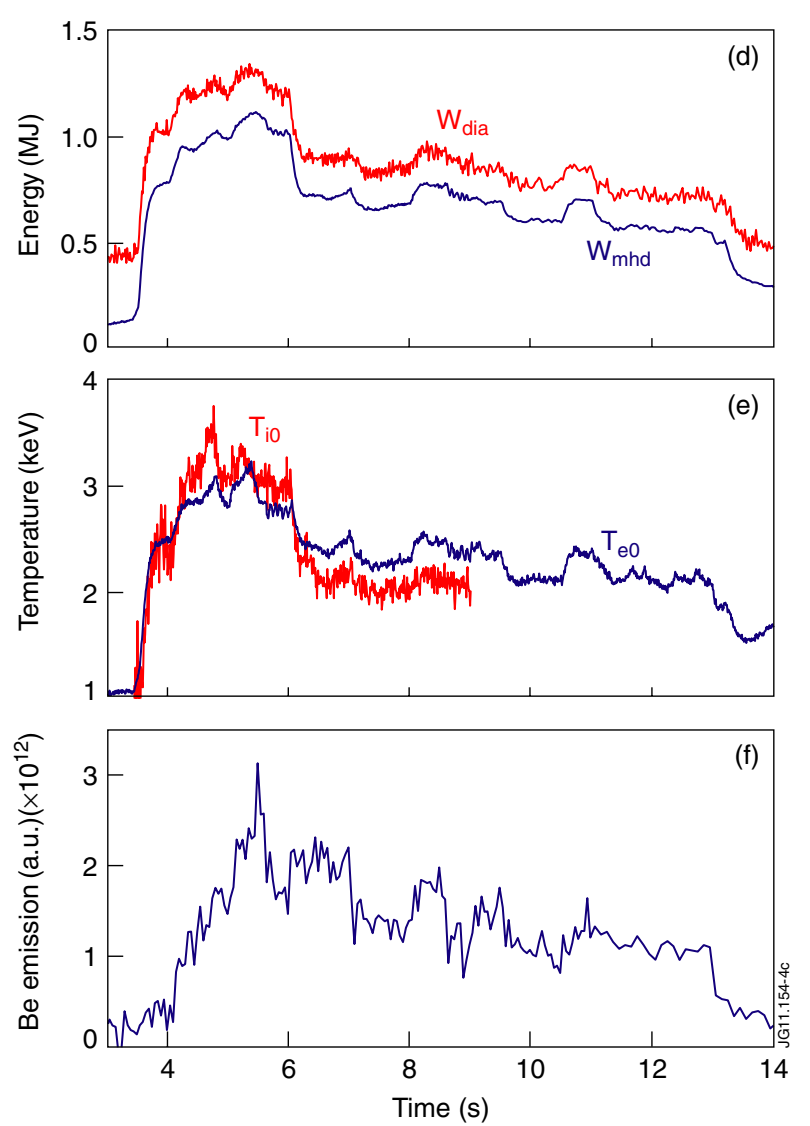

Figure 4. Typical discharge of the fundamental H ICRH experiments (JPN 79335): (a) ICRF and NBI power, $(b)$ radiated power, $(c)$ central line-integrated density, $(d)$ diamagnetic and equilibrium plasma energies, $(e)$ electron $(\mathrm{ECE})$ and ion $(\mathrm{CXRS})$ central temperatures, $(f)$ beryllium line emission intensity.

suggestions for further investigation for the optimization of the ICRH heating scenarios available for the non-active phase of ITER at reduced magnetic fields.

\section{Fundamental H majority ICRF heating-experimental results}

\subsection{Overview}

The study of the fundamental hydrogen ICRF heating scheme in $\mathrm{H}$ majority plasmas was performed at $B_{0}=2.65 \mathrm{~T}$ and $f=42.5 \mathrm{MHz}$ with dipole antenna phasing $(0 \pi 0 \pi)$. Up to $5.5 \mathrm{MW}$ of ICRF power was coupled to the plasma. In figure 4 several time traces of a typical discharge of these experiments are represented: ( $a$ ) ICRF and NBI power, $(b)$ total radiated power (bolometer), $(c)$ central line-integrated density (interferometer), (d) diamagnetic $\left(W_{\text {dia }}\right)$ and equilibrium ( $\left.W_{\text {mhd }}\right)$ plasma energies (from EFIT), $(e)$ electron (ECE) and ion (CXRS) central temperatures, $(f)$ beryllium $257 \mathrm{~nm}$ line emission intensity (visible edge spectroscopy).

The RF power steps seen in figure $4(a)$ (at $t=7 \mathrm{~s}$ and $t=9.5 \mathrm{~s}$ ) were applied in practically all discharges for diagnostic purposes. It is already interesting to note that, when removing $2.5 \mathrm{MW}$ of the applied RF power (at e.g. $t=7 \mathrm{~s}$ ), the electron and ion temperatures $(e)$ drop only by about $0.3 \mathrm{keV}$ indicating that this is not a very efficient heating scheme. The radiated power $(b)$, which drops by approximately $1 \mathrm{MW}$ and the clear reduction in the Be impurity level $(f)$ during the ICRF power confirm well the relatively poor efficiency of this heating scenario.

\subsection{Power absorption and heating efficiency}

By analysing the response of the plasma energy to the ICRF power steps it is possible to infer the heating efficiency of the ICRH scenario, defined as $\eta=P_{\mathrm{abs}} / P_{\mathrm{RF}}$. Here, $P_{\mathrm{RF}}$ is the total RF power coupled to the plasma (computed directly from RF measurements in the antenna circuit) and $P_{\text {abs }}$ is the power actually absorbed in the plasma. The latter can be determined either by analysing the global diamagnetic/equilibrium energy response of the plasma or by integrating the power absorption profiles obtained by the individual analysis of the electron and ion temperature responses to the change in the RF power steps (local power absorption). The main difference between the two techniques is that the plasma energy measurements include, to some extent, the contribution of the suprathermal ions accelerated by ICRH whereas the temperature measurements (by ECE and by charge-exchange) include only the contribution of the thermal particles and of the fast ions that had already time to transfer their energy to the bulk plasma. As long as the steady-state fast ion population is small (which is typically the case in majority ICRF heating schemes), the two ways of computing the heating efficiency give similar results. 

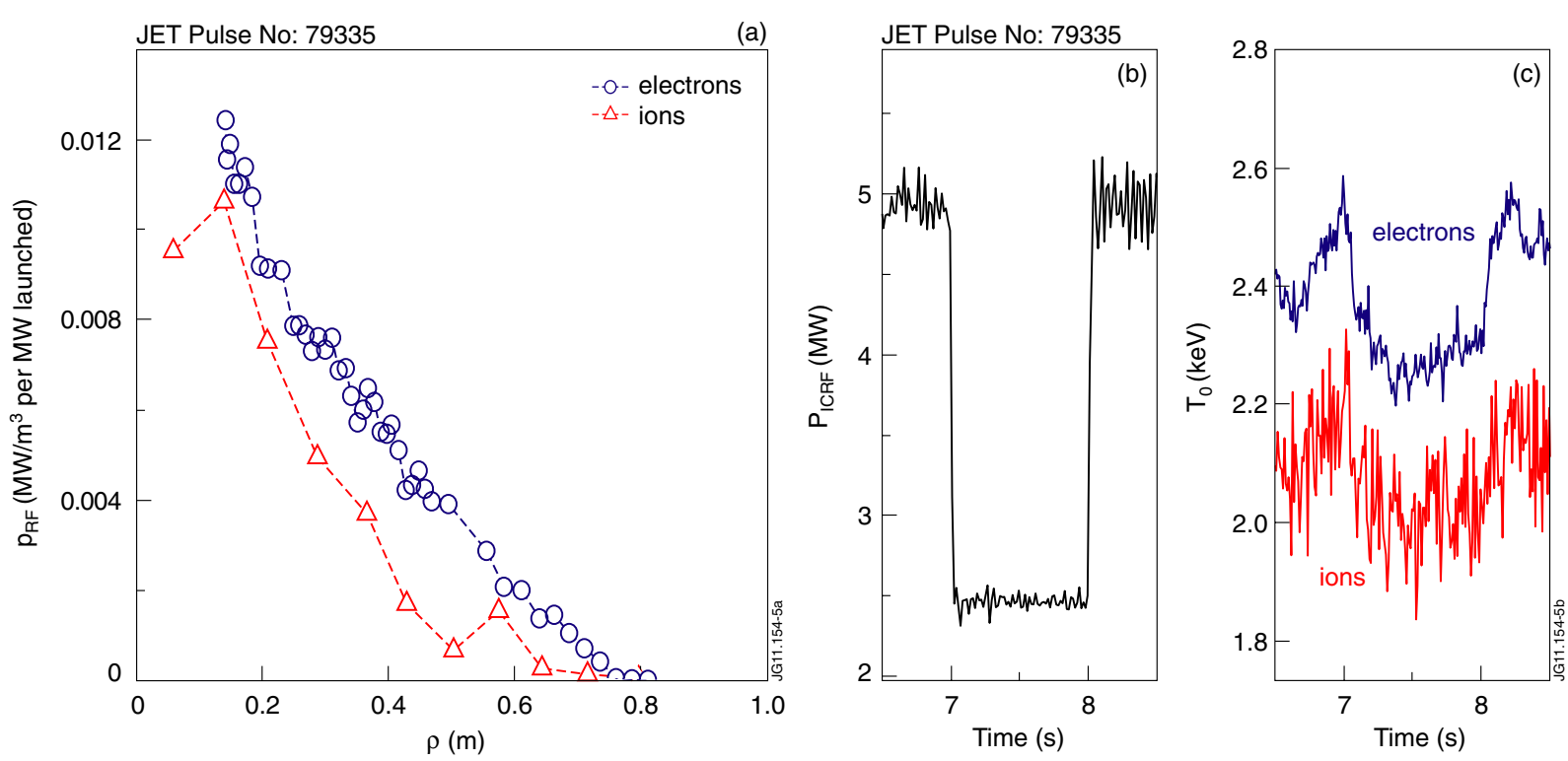

Figure 5. (a) ICRF power absorption profiles for ions (circles) and electrons (triangles) computed by BIS analysis of the CXRS and ECE signals during a 2.5 MW RF power step in pulse 79335. (b), (c) Example of the ICRF power step and central ion/electron temperature responses used to compute the power absorption profiles.

In figure 5(a) the power absorption profiles for electrons (circles) and ions (triangles) computed, respectively, by an exponential break-in-slope (BIS) analysis [19] of the ECE and CXRS signal responses to the RF power step in pulse \#79335 at $t=7 \mathrm{~s}$ (figures $5(b)$ and $(c)$ ) are represented. Similar profiles are obtained when analysing the RF turn-on step at $t=8 \mathrm{~s}$.

When integrating the power absorption profiles shown in figure 5(a), only about one-third of the RF power coupled to the plasma is recovered, confirming the low heating efficiencies expected for this scenario. It was observed, however, that the heating efficiency is improved when operating at higher plasma temperatures. This is illustrated in figure $6(a)$, where the heating efficiencies for ions (squares) and electrons (circles) computed by integrating the power absorption profiles inferred by BIS analysis of the CX and ECE signals are represented as a function of the plasma central temperature averaged around the break instants. The data points, collected from various discharges, were obtained with ICRF power steps of about $2.5 \mathrm{MW}$ with constant NBI power (1.3 MW) and similar plasma density. The different plasma temperatures come from the different pre-heating levels applied before the instants considered for the BIS analyses. Note that the heating efficiencies of both ions and electrons increase with the bulk plasma temperature, reaching a total value of $\eta=0.4$ for the maximum temperature achieved in the experiments $\left(T_{\mathrm{e}}=2.55 \mathrm{keV}\right)$. The slope of the heating efficiency of the ions is somewhat steeper than the one for the electrons, indicating that the ion cyclotron absorption of the $\mathrm{H}$ ions is privileged over electron Landau damping when increasing the bulk plasma temperature within the studied range. The enhancement of ion absorption with plasma temperature is actually expected from basic wave theory and is related to the broadening of the ion cyclotron resonance absorption region at higher temperatures.

In figure $6(b)$, the total ICRF heating efficiency values $\eta=\eta_{\mathrm{e}}+\eta_{\mathrm{i}}$, computed as the sum of the electron and ion contributions, shown in figure 6(a) (triangles), are compared with the heating efficiencies inferred by analysing the plasma energy responses to the RF power variations (circles). The latter were computed at the same RF power steps as the $T_{\mathrm{e}} / T_{\mathrm{i}}$ BIS data. The good agreement between the results of the two methods shows that the fast particle contribution to the energy balance of the plasma is small under these conditions, suggesting that the $\mathrm{RF}$ acceleration of the $\mathrm{H}$ minority is modest.

\subsection{Fast particles}

A more detailed assessment of the creation of supra-thermal populations in these experiments was carried out using the NPAs. In figure 7(a), the response of several channels of the horizontal NPA (KR2) during a drop in the ICRF power is shown. The $\mathrm{H}$ ion energies corresponding to the different NPA channels plotted are indicated in the legend. Figure 7(b) illustrates the distribution functions of the $\mathrm{H}$ ions (below $30 \mathrm{keV}$ ) for $P_{\mathrm{RF}}=2.5 \mathrm{MW}$ (circles) and $P_{\mathrm{RF}}=5.0 \mathrm{MW}$ (triangles) obtained by integrating the NPA fluxes during $\Delta t=9.6-10.4 \mathrm{~s}$ and $\Delta t=10.6-11.4 \mathrm{~s}$, respectively. The effect of ICRF absorption on the $\mathrm{H}$ ion distribution is evident: the number of $\mathrm{H}$ ions with energies around $20-30 \mathrm{keV}$ is almost one order of magnitude higher when $5 \mathrm{MW}$ (instead of 2.5 MW) of ICRF power is applied to the plasma. The effective temperatures of the supra-thermal $\mathrm{H}$ population estimated by a linear fit of the log-scale slope of the data points with $E>10 \mathrm{keV}$ are $T_{\text {eff }}=4.2 \mathrm{keV}$ and $T_{\text {eff }}=5.7 \mathrm{keV}$ for the $P_{\mathrm{RF}}=2.5 \mathrm{MW}$ and $P_{\mathrm{RF}}=5 \mathrm{MW}$ time intervals, respectively. The central ion temperatures measured by CXRS are only about $T_{\mathrm{i} 0}=2 \mathrm{keV}$ and $T_{\mathrm{i} 0}=2.3 \mathrm{keV}$, respectively.

Although the effect of the ICRF power on the $\mathrm{H}$ ion distribution is visible in the NPA measurements, it is important to recall that the $\mathrm{H}$ ion tails observed in these experiments are very small when compared with the typical RF-driven tails measured in minority heating experiments, which can 

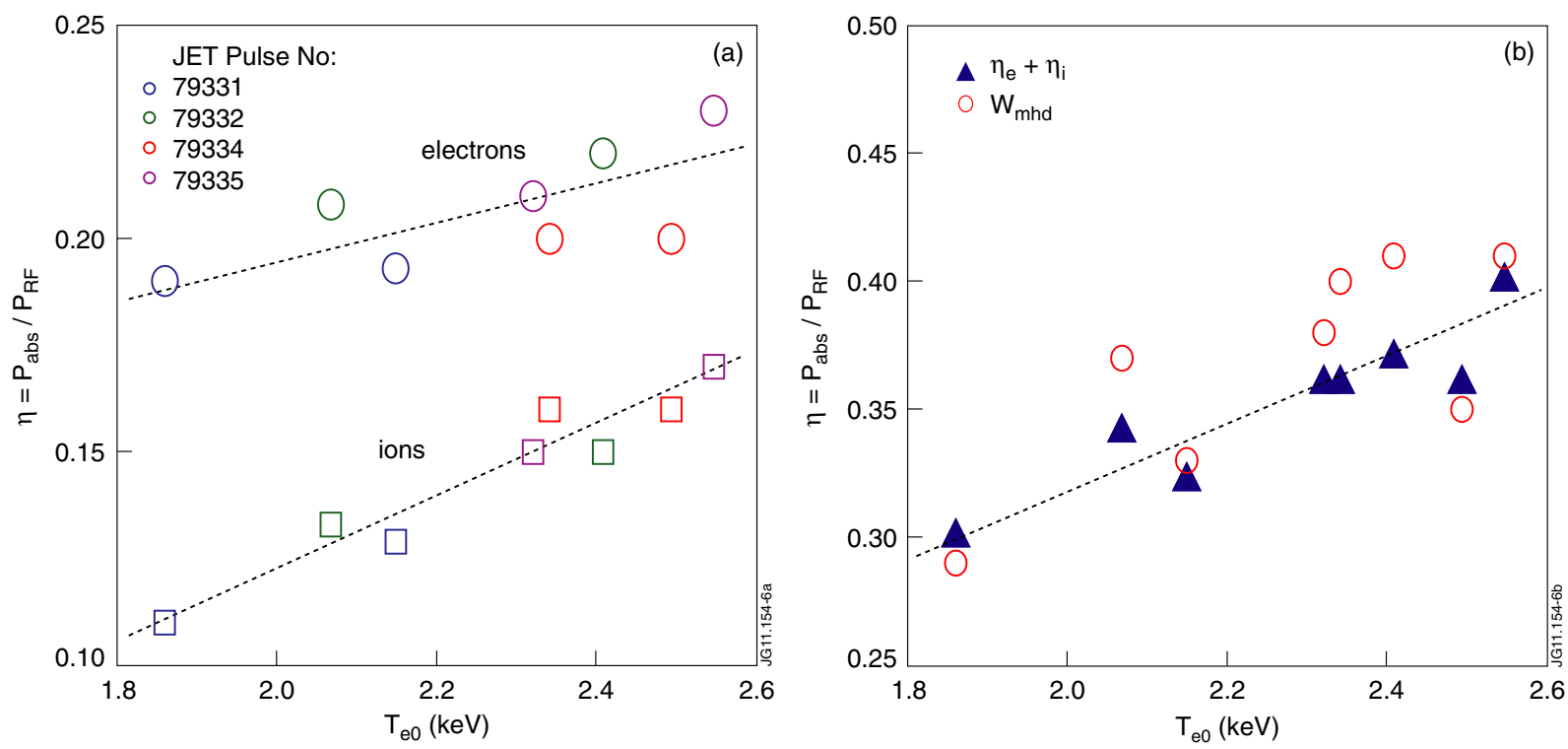

Figure 6. ( $a$ ) Heating efficiencies obtained for ion (squares) and for electrons (circles) by integrating the power absorption profiles inferred from BIS analysis of the CXRS and ECE signals as a function of the plasma central temperature. (b) Comparison between the total heating efficiency obtained by summing the individual species' contributions (triangles) with the results obtained by analysing the global plasma energy response ( $\left.W_{\mathrm{mhd}}\right)$ to the ICRF power steps (circles).
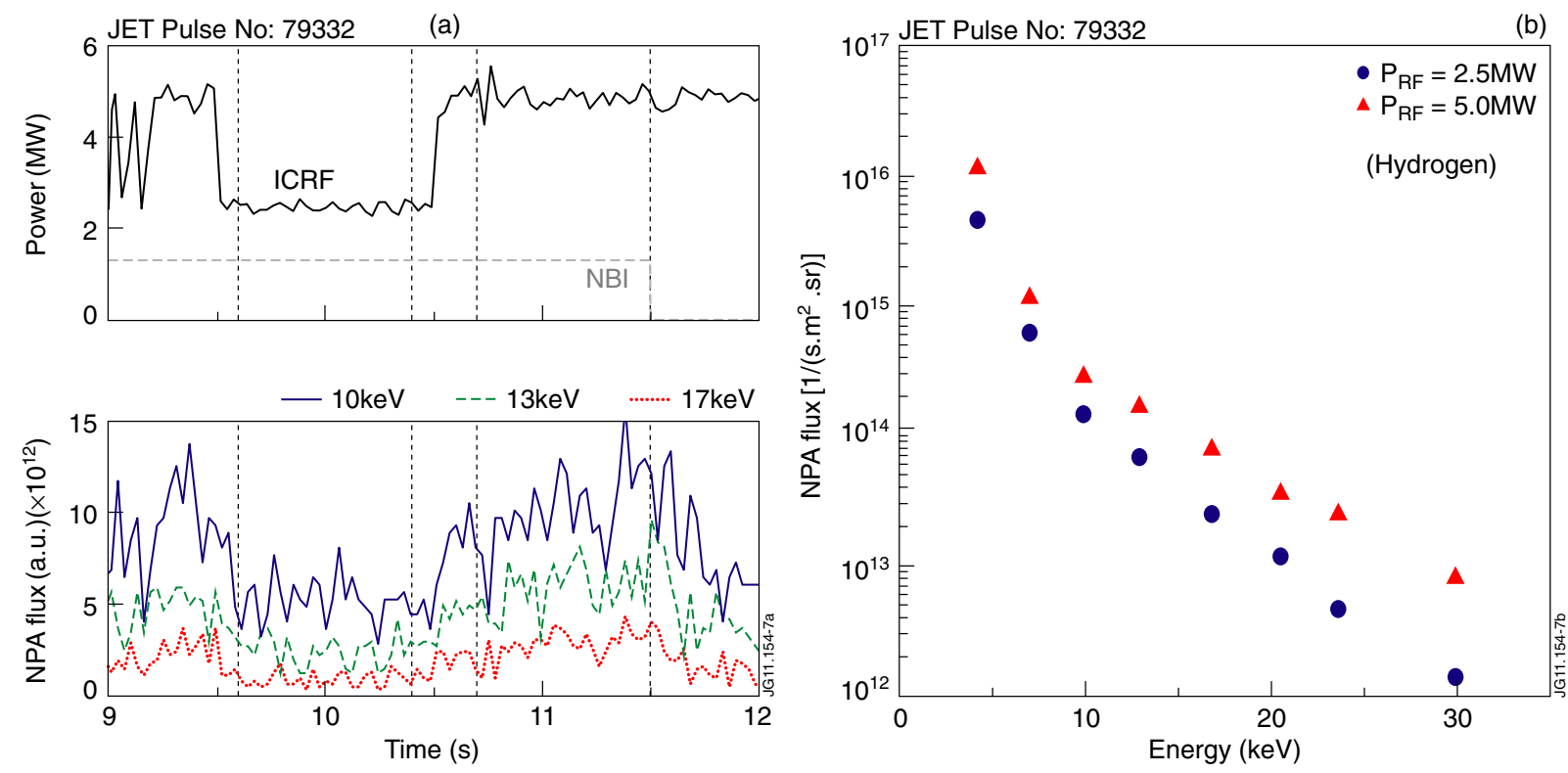

Figure 7. (a) Response of three channels of the horizontal NPA to a power well imposed in the ICRF power. $(b)$ Distribution function of the $\mathrm{H}$ ions obtained by integrating the NPA signals during the $P_{\mathrm{RF}}=2.5 \mathrm{MW}$ (circles) and during the $P_{\mathrm{RF}}=5 \mathrm{MW}$ (triangles) RF power phases. The integration time was $0.8 \mathrm{~s}$ in both cases.

reach the $\mathrm{MeV}$ energy range (with effective temperatures of a few hundred $\mathrm{keV}$ ). In addition, unlike in standard $\mathrm{H}$ minority heating cases, the fast ion losses detected with the scintillator probe (KA3) were negligible throughout the session. The modest ion acceleration observed in the fundamental $\mathrm{H}$ majority experiments is expected from theory due to the combination of the low ion absorption close to the fundamental resonance and the large concentration of $\mathrm{H}$ ions that share the local power density available.

Apart from the expected power dependence, the RF acceleration of the $\mathrm{H}$ ions is also dependent on the plasma collisionality. The slowing-down time $\tau_{\mathrm{s}}(\mathrm{s})=$ $0.133\left(A_{\mathrm{i}} / Z_{\mathrm{i}}^{2}\right) T_{\mathrm{e}}^{3 / 2} / n_{\mathrm{e}}$ over which the fast ions transfer most of their energy to the bulk plasma [20] gives a measure of the collisionality effect. In this expression, $A_{\mathrm{i}}$ and $Z_{\mathrm{i}}$ are, respectively, the mass and charge numbers of the examined ion species while $T_{\mathrm{e}}$ and $n_{\mathrm{e}}$ represent the electron plasma temperature and density (given here in $\mathrm{keV}$ and $10^{19} \mathrm{~m}^{-3}$, respectively). Therefore, for a given RF power, one would expect to observe an enhancement of the fast ion tail formation for higher temperatures and/or for lower densities of the plasma. 


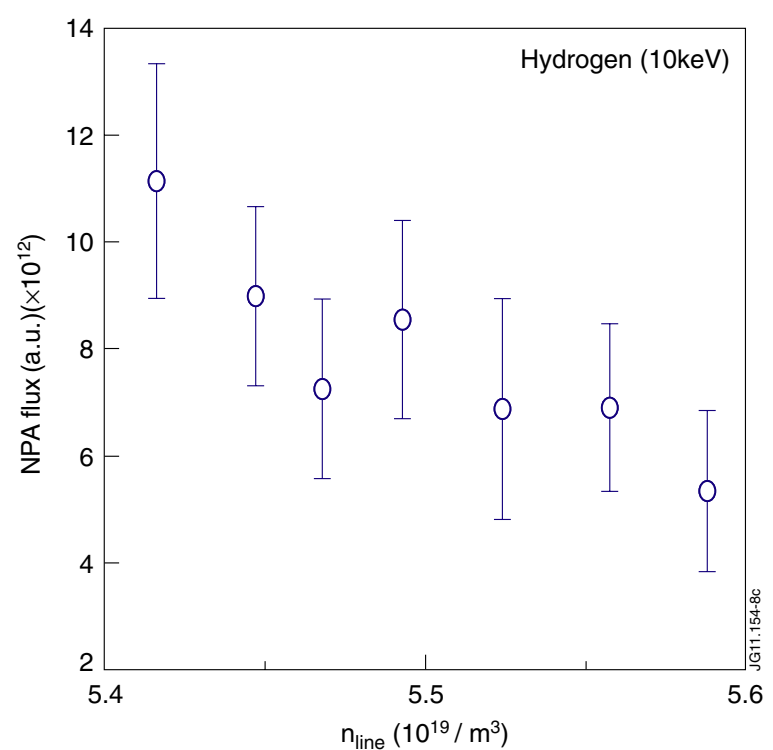

Figure 8. Averaged values of the horizontal NPA fluxes for $10 \mathrm{keV}$ $\mathrm{H}$ ions as a function of the central line-integrated plasma density.

This dependence was verified experimentally as illustrated in figure 8, where the averaged NPA fluxes representing the $10 \mathrm{keV} \mathrm{H}$ ions measured with the horizontal NPA (KR2) are plotted against the central (line-integrated) plasma density $n_{\text {line }}$. The data points represent average values taken in $\Delta t=$ $0.2 \mathrm{~s}$ time windows in several pulses with similar input powers: $P_{\mathrm{ICRH}}=4.9 \pm 0.1 \mathrm{MW}$ and $P_{\mathrm{NBI}}=1.3 \mathrm{MW}$. The error bars represent the standard deviation of the averaged values. The plasma temperature was practically the same in all intervals considered, $T_{\mathrm{e} 0}=2.35 \pm 0.05 \mathrm{keV}$. Note that by reducing the plasma density by only about $0.2 \times 10^{19} \mathrm{~m}^{-3}$, the NPA fluxes are reduced by roughly a factor of two in the studied energy range. A more detailed analysis based on the NPA energy spectra at different densities shows that the effective temperature of the supra-thermal ions (proportional to the slope of the distribution) is not strongly modified in the density range studied, suggesting that the decrease observed in the NPA flux at higher densities is a pure collisional effect rather than related to a change in the ICRF absorption.

The temperature dependence of the RF acceleration of the $\mathrm{H}$ ions is depicted in figure 9, where the averaged NPA fluxes corresponding to the $10 \mathrm{keV}$ horizontal NPA channel are plotted against the central plasma temperature $T_{\mathrm{e} 0}$ measured by fast ECE (at $R=3.11 \mathrm{~m}$ ). The data points represent $200 \mathrm{~ms}$ time intervals with $P_{\mathrm{NBI}}=1.3 \mathrm{MW}$ (circles) and $P_{\mathrm{NBI}}=2.6 \mathrm{MW}$ (triangles) NBI power but with the same RF power level, $P_{\mathrm{ICRH}}=4.9 \pm 0.2 \mathrm{MW}$. The plasma densities were nearly constant in all the time intervals considered.

Note that the neutral flux related to the $10 \mathrm{keV} \mathrm{H}$ ions is increased by a factor of roughly two when the plasma temperature is increased from $T_{\mathrm{e}} \approx 2.2 \mathrm{keV}$ to $T_{\mathrm{e}} \approx 2.5 \mathrm{keV}$. Unlike the density dependence, the increase in the NPA fluxes observed for higher plasma temperatures (at constant ICRF power) is not only due to the decreased plasma collisionality, but is also associated with the enhanced ICRF absorption efficiency observed at higher temperatures, as discussed in

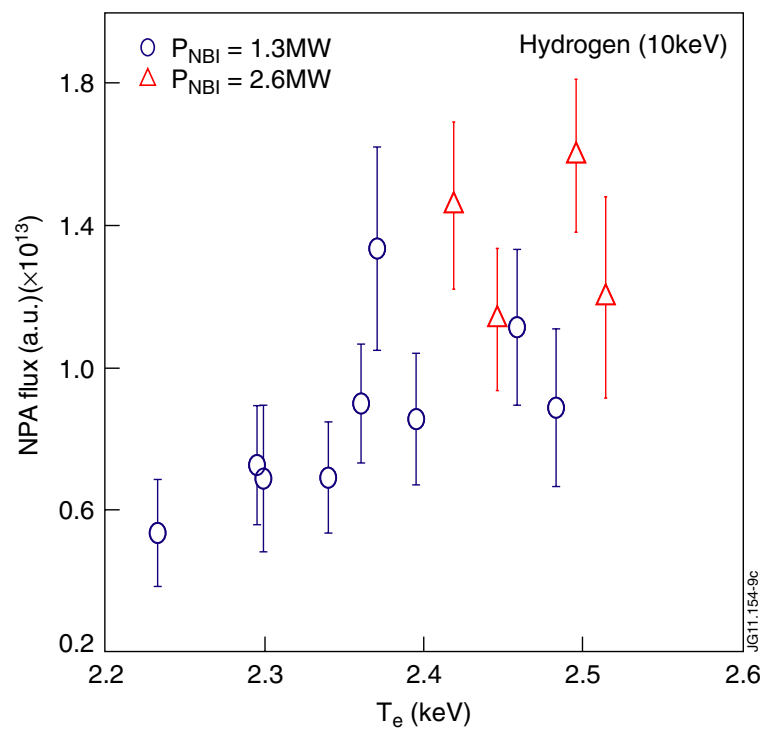

Figure 9. Time-averaged values $(\Delta t=200 \mathrm{~ms})$ of the $10 \mathrm{keV}$ horizontal NPA signal as a function of the central plasma temperature. The circles represent instants with $1.3 \mathrm{MW}$ while the triangles represent instants with $2.6 \mathrm{MW}$ of NBI power.

section 2.2 (figure 6). The relative importance of these two processes on the formation of the supra-thermal $\mathrm{H}$ population is difficult to quantify without complex wave/Fokker-Planck numerical modelling. Preliminary attempts using a simple 1D Fokker-Planck code [21] fed with RF power densities found in the experiments at low and high $T_{\mathrm{e}}$ indicate that the enhancement of the RF absorptivity with the plasma temperature contributes with roughly $1 / 3$ to the acceleration of the $\mathrm{H}$ ions in the $10 \mathrm{keV}$ energy range, the other $2 / 3$ being related to the change in the plasma collisionality.

The vertical NPA (KF1), with line-of-sight close to the radial location of the fundamental $\mathrm{H}$ ion cyclotron resonance layer $(R=3.15 \mathrm{~m})$, was set to monitor $\mathrm{H}$ ions with energies between 140 and $550 \mathrm{keV}$. For $P_{\mathrm{NBI}}<5 \mathrm{MW}$, the number of counts in the KF1 channels was negligible, independent of the ICRF power applied. When more than $5 \mathrm{MW}$ NBI power was applied to the plasma, hints of $\mathrm{H}$ ions with energies up to $\sim 200 \mathrm{keV}$ were observed, but the number of counts was still too modest for a relevant statistical analysis of the distribution function in the high-energy region. This is illustrated in figure 10, where the signals of the four lowest energy channels of the vertical NPA are shown during the first half of pulse 79332, where high-power ICRF and NBI were applied simultaneously $(a)$.

Even with a limited number of counts, the response of the lowest energy NPA signals ( $E<228 \mathrm{keV}$ ) to the ICRF power during the high-power NBI phase is clear: When raising the ICRF power from 2.5 to $5 \mathrm{MW}(t=6 \mathrm{~s})$ the neutral counts begin to increase up to the starting instant of the RF power modulation phase $(t=7 \mathrm{~s})$, where the average power is only about $P_{\mathrm{RF}}=3.7 \mathrm{MW}$ and the NPA signals decrease. After the RF power is switched back to $5 \mathrm{MW}(t=7.4 \mathrm{~s})$ the number of counts starts increasing again, with a similar slope as in the previous $P_{\mathrm{RF}}=5 \mathrm{MW}$ phase. The fact that the response of the neutral counts representing the high-energy $\mathrm{H}$ ions to the 


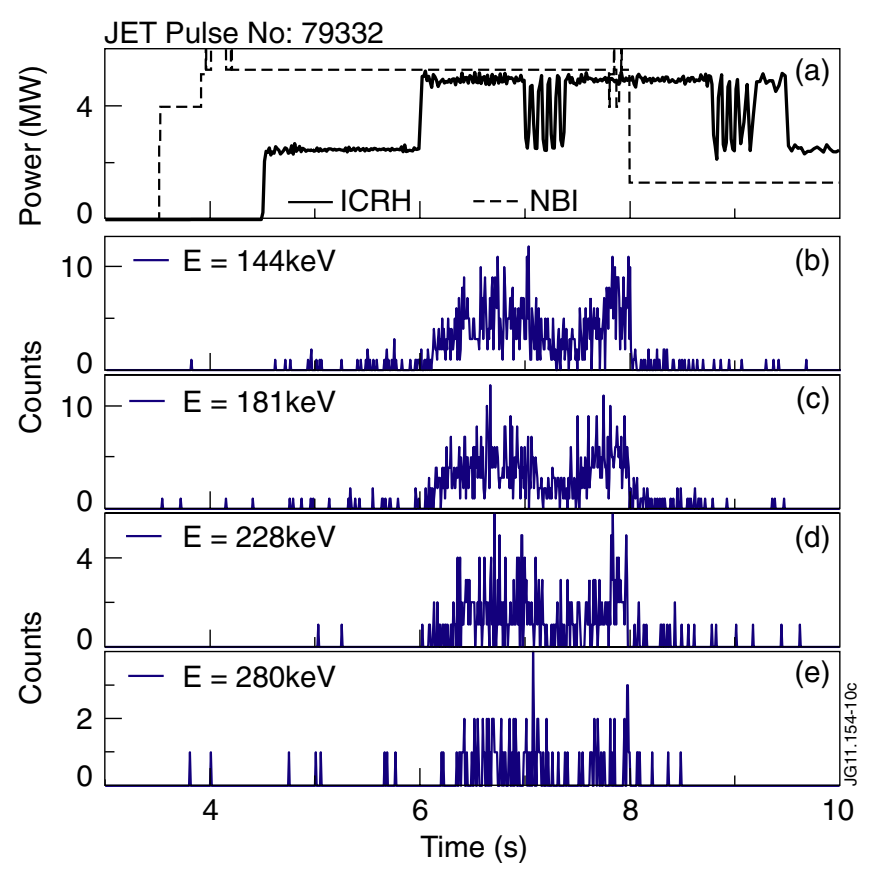

Figure 10. (a) ICRF and NBI power waveforms in pulse 79332. (b)-(e) Raw signals of the first four energy channels of the vertical NPA (KF1), sampling ion energies from 140 to $280 \mathrm{keV}$.

RF power steps is rather slow illustrates once again the strong competition between the weak RF acceleration of the majority $\mathrm{H}$ ions with the collisional drag of the plasma, in particular in this relatively high energy range. The number of counts of the NPA channels for $E=280 \mathrm{keV}(e)$ is negligible even during the high-power phase.

The fact that $\mathrm{H}$ ions with energies around $200 \mathrm{keV}$ are only detected when combined $5 \mathrm{MW} \mathrm{RF}+5 \mathrm{MW}$ NBI power is applied to the plasma and not when $5 \mathrm{MW}$ RF alone (+1.3 MW $\mathrm{NBI}$ ) is applied is not related to $N=2 \mathrm{ICRF}$ absorption of the NBI D ions, as will be discussed in the next paragraph. A possible explanation for the higher fraction of fast $\mathrm{H}$ ions observed with $10 \mathrm{MW}$ of auxiliary power input is simply the fact that in this phase the plasma temperature is relatively high ( $T_{\mathrm{e}} \approx T_{\mathrm{i}} \approx 3.5 \mathrm{keV}$ ) and the collisionality of the plasma is somewhat lower than that in the phases of the discharge where less auxiliary power is injected. These results suggest once again that operating at higher plasma temperatures is beneficial for the fundamental $\mathrm{H}$ majority heating scheme, because of the lower collisionality of the plasma or because of the enhanced ICRF absorption efficiency or both.

As mentioned previously, second harmonic absorption of fast D ions (injected by NBI) could also take place in this heating scenario, despite the off-axis/low-energy PINIs chosen to minimize this effect. The horizontal NPA, set for D energies below $50 \mathrm{keV}$, sampled the lower part of the slowingdown beam distribution function (including the thermalized population) and did not show any significant variation of the neutral fluxes with ICRF power for any level of NBI power applied, indicating that RF acceleration in this region of phase space was negligible. The vertical NPA, which was set to monitor the RF acceleration of D-beam ions above the beam source energy $\left(130 \mathrm{keV}<E_{\mathrm{D}}<500 \mathrm{keV}\right)$ in some of the discharges, did not register any counts at all. The absence of fast $\mathrm{D}$ ions above the beam source energies $\left(E_{\mathrm{NBI}} \approx 80 \mathrm{keV}\right)$ was also confirmed by measurements with the neutron spectrometer TOFOR, which found D distributions consistent with beam-beam ion reaction only, independent of the amount of NBI or ICRF power applied. Gamma-ray spectroscopy further confirmed the total absence of fast $\mathrm{D}$ ions in these experiments.

It is important to mention once again that the conditions of the experiments were chosen to minimize the parasitic D absorption. Therefore, the absence of fast $\mathrm{D}$ ions confirmed by various diagnostics only corroborates the 'good' choice of the plasma parameters and NBI settings to reduce this process and does not suggest, by any means, that harmonic D absorption cannot play an important role in future experiments (with different PINI choices) or in the case of $\mathrm{D}$ beams being applied to the $\mathrm{H}$ plasmas in ITER.

\subsection{Global performance and confinement}

The study of the overall heating performance of the $\mathrm{H}$ majority discharges was carried out by a statistical analysis of the average values of the main diagnostic signals in multiple time intervals sampled throughout various plasma discharges. For each of the five pulses included in the analysis, 10 time intervals of $0.2 \mathrm{~s}$ were considered for the averages and the resulting data points were divided into subclasses according to the NBI power level applied. The data shown in the previous subsection when discussing the dependence of the NPA results to the plasma density and temperature were already part of this data set. In this subsection, the dependences of several plasma parameters with the ICRF power will be presented.

In figure 11 , the averaged values of a few key plasma parameters are represented as a function of the (averaged) ICRF power applied: $(a)$ central electron temperature, $(b)$ central ion temperature, $(c)$ total radiated power and $(d)$ energy confinement time. The circles represent intervals with $P_{\mathrm{NBI}}=$ 1.3 MW (1 PINI) while the triangles correspond to intervals with $P_{\mathrm{NBI}}=2.6 \mathrm{MW}$ ( 2 PINIs). The values at $P_{\mathrm{ICRH}}=0$ represent the different averaged quantities before the ICRF power was applied to the discharges. The plasma densities (not shown) were similar in the time intervals with 1 and with 2 NBI PINIs but showed a small but systematic increase with the ICRF power, varying from $n_{\text {line }}=(5.40 \pm 0.05) \times 10^{19} \mathrm{~m}^{-3}$ at low RF power to $n_{\text {line }}=(5.70 \pm 0.05) \times 10^{19} \mathrm{~m}^{-3}$ at $5 \mathrm{MW}$. The error bars indicate the standard deviation of the average values in each time interval considered.

Both the electron and ion temperatures show a roughly linear increase with the ICRF power applied, although hints of a 'saturation' tendency on the $T_{\mathrm{i}}$ values could be interpreted. The slopes indicated in figures $11(a)$ and $(b)$ are $\Delta T_{\mathrm{e}, \mathrm{i}} / \Delta P_{\mathrm{RF}}=$ $0.1 \mathrm{keV} \mathrm{MW}^{-1}$ for both species, and correspond to the linear fit of the data points with $P_{\mathrm{NBI}}=1.3 \mathrm{MW}$ (discarding the point with $\left.P_{\mathrm{RF}}=0\right)$ in both cases. Although the data with higher NBI power (2.6 MW) are too scarce for a meaningful linear fit, they show a similar tendency. Furthermore, it is interesting to note that the average plasma temperatures are increased by only about $0.2-0.3 \mathrm{keV}$ when $1.3 \mathrm{MW}$ of additional NBI 

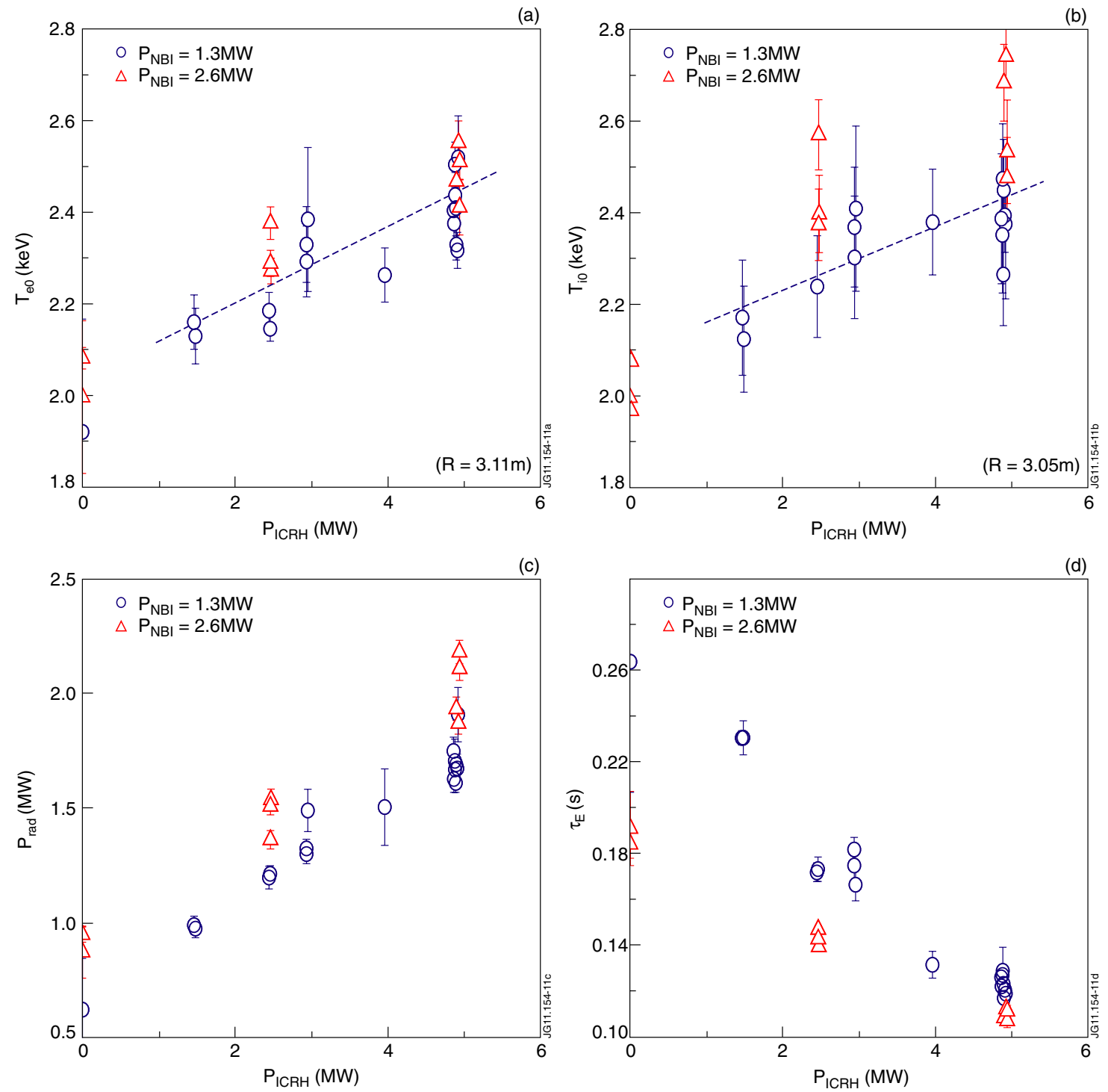

Figure 11. Averaged values of several signals as a function of the ICRF power during $0.2 \mathrm{~s}$ time intervals sampled in various discharges with $1.3 \mathrm{MW}$ (circles) and $2.6 \mathrm{MW}$ (triangles) NBI power: $(a)$ central electron temperature (ECE at $R=3.11 \mathrm{~m}$ ); $(b)$ central ion temperature (CXRS at $R=3.05 \mathrm{~m}) ;(c)$ total radiated power (bolometer); $(d)$ energy confinement time.

power is applied. It is important to recall that these values only represent the 'steady-state' temperatures achieved in the discharges after adding a certain amount of ICRF power to the original NBI-only plasma (with $T_{\mathrm{e}} \approx T_{\mathrm{i}} \approx 2.0 \mathrm{keV}$ ) and should not be confused with the RF heating efficiencies determined by analysing the fast response of the plasma energy to ICRF power changes presented in section 2.2, where the transport effects are minimized. The low values of the $\Delta T / \Delta P_{\mathrm{RF}}$ slopes are not only a consequence of the poor ICRF absorptivity of the fundamental $\mathrm{H}$ majority heating scenario but are also related to the relatively high radiation losses caused by enhanced plasma-wall interaction (see section 4) and poor confinement of the heated hydrogen majority plasmas, as illustrated, respectively, in figures $11(c)$ and $(d)$. Note that when $5 \mathrm{MW}$ of ICRF power is added to the original plasma the total radiation losses increase by approximately $1.2 \mathrm{MW}$, suggesting that about $1 / 4$ of the RF power launched is lost by radiation. The confinement time is reduced by a factor of about two when $5 \mathrm{MW}$ of ICRF power is applied. It is interesting to note that the confinement deterioration is worse for the low power than for the higher power NBI pre-heating case, where the original plasmas (before ICRF) already exhibit a significantly lower confinement. A more detailed assessment of the power balance of these discharges will be made in section 5 .

\subsection{Plasma dilution}

As mentioned before, traces of ${ }^{4} \mathrm{He}$ ions were present in all the pulses of the $\mathrm{H}$ majority heating session. The ${ }^{4} \mathrm{He}$ concentration was not controlled explicitly but varied 'randomly' during the experiments depending on the level of NBI pre-heating chosen (as these experiments followed experiments using ${ }^{4} \mathrm{He}$ beams, ${ }^{4} \mathrm{He}$ ions trapped in the beam line ion dumps were released for some time during subsequent operation with D beams). Using the statistical 

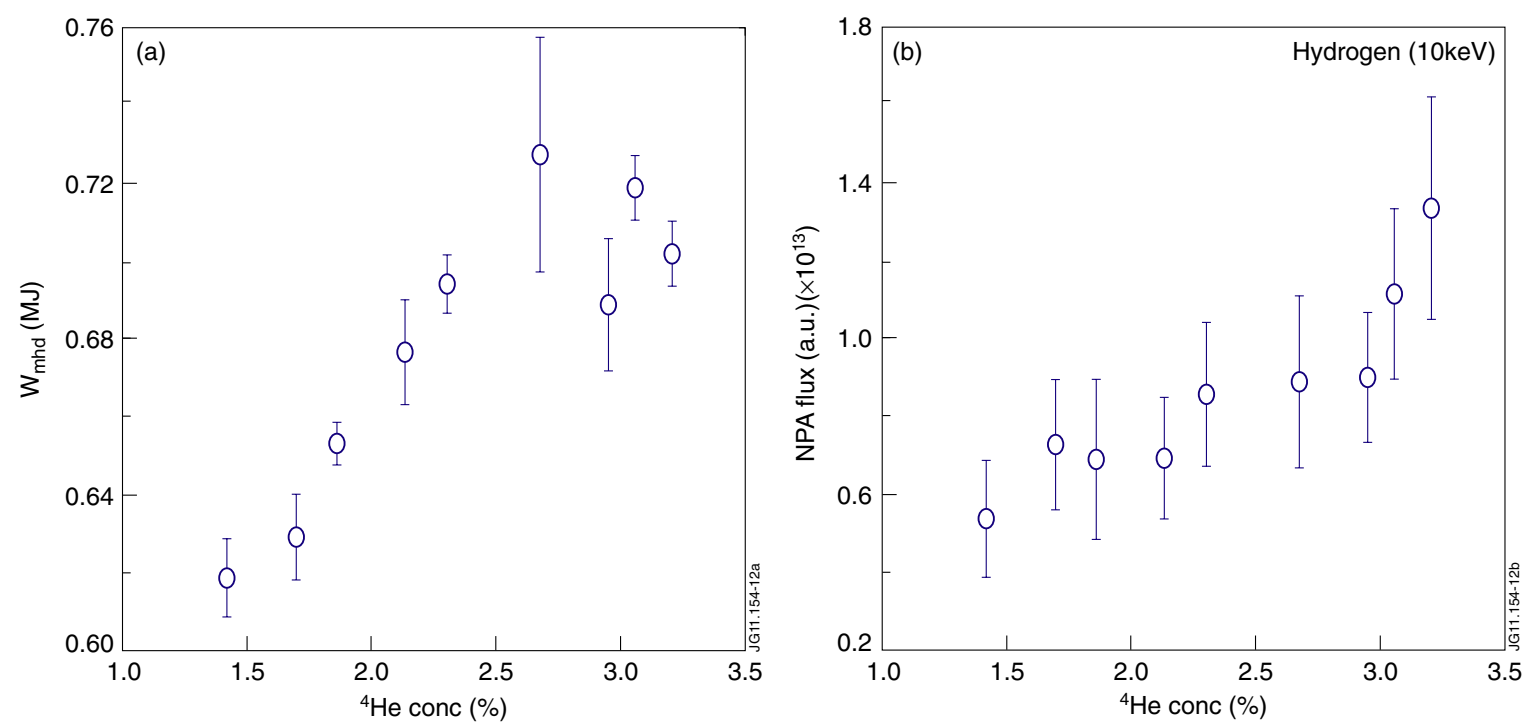

Figure 12. (a) Averaged values of the plasma equilibrium energy ( $\left.W_{\mathrm{MHD}}\right)$ as a function of the ${ }^{4} \mathrm{He}$ concentration for fixed auxiliary input power $\left(P_{\mathrm{RF}}=4.9 \mathrm{MW}, P_{\mathrm{NBI}}=1.3 \mathrm{MW}\right)$. $(b)$ Averaged NPA fluxes $(\mathrm{KR} 2)$ for the $10 \mathrm{keV} \mathrm{H}$ ions as a function of the ${ }^{4} \mathrm{He}$ concentration for the same time intervals as considered on the left.

analysis based on the time-averaged quantities described in the previous subsection, evidence of improved ICRF heating efficiency for higher ${ }^{4} \mathrm{He}$ fractions present in the plasma was observed. This is illustrated in figure $12(a)$, where the equilibrium plasma energies $\left(W_{\text {mhd }}\right)$ averaged over $0.4 \mathrm{~s}$ time windows in various pulses are plotted against the corresponding averaged ${ }^{4} \mathrm{He}$ concentrations, estimated via edge spectroscopy measurements (the ${ }^{4} \mathrm{He}$ concentration was calculated by the real-time ${ }^{3} \mathrm{He}$ injection system [7], which was calibrated during this particular session). All the data points correspond to similar plasma densities, $n_{\text {line }}=(5.5 \pm$ $0.1) \times 10^{19} \mathrm{~m}^{-3}$, NBI power levels (1.3 MW) and ICRF power applied $P_{\mathrm{RF}}=4.9 \pm 0.1 \mathrm{MW}$. As for the equilibrium $W_{\mathrm{mhd}}$ plasma energy, the electron and ion temperatures (not shown) are systematically enhanced for higher ${ }^{4} \mathrm{He}$ concentrations (the plasma density values did not show any clear correlation with the ${ }^{4} \mathrm{He}$ concentration in the represented time intervals).

In figure $12(b)$, the neutral fluxes corresponding to the $E=10 \mathrm{keV}$ NPA channel (averaged over the same time intervals as the data in figure 12(a)) are represented, indicating that the increased values of the plasma energy achieved at higher ${ }^{4} \mathrm{He}$ concentrations are due to the enhancement of the $\mathrm{H}$ ion cyclotron absorption rather than to the possible $N=2$ harmonic absorption of the ${ }^{4} \mathrm{He}$ ions. The confinement time and radiated power are practically the same in all the time instants indicating that the overall power balance does not explicitly depend on the ${ }^{4} \mathrm{He}$ concentration within the studied range. One possible explanation for the enhancement of the heating performance for higher ${ }^{4} \mathrm{He}$ concentrations is the eventual change in the RF field polarization when diluting the $\mathrm{H}$ plasma with an additional species [22]. Although preliminary simulations indicate that this effect should be small for small ${ }^{4} \mathrm{He}$ (or D) concentrations, this experimental evidence should be explored more carefully (both numerically and in future experiments) since it could help to increase the efficiency of this ICRF scenario and thus have an impact on the initial operation of ITER.

\section{Second harmonic $(N=2)^{3} \mathrm{He}$ ICRF heating-experimental results}

\subsection{Overview}

The study of the second harmonic ${ }^{3} \mathrm{He}$ ICRF heating scheme in $\mathrm{H}$ majority plasmas was performed at $B_{0}=2.65 \mathrm{~T}$ and $f=51.5 \mathrm{MHz}$ using dipole antenna phasing. The plasma temperature and densities were similar to those in the fundamental $\mathrm{H}$ majority experiments: $n_{0} \approx 3 \times 10^{19} \mathrm{~m}^{-3}$ and $T_{0}=2-4 \mathrm{keV}$, depending on the NBI and RF power levels applied. Up to $5.5 \mathrm{MW}$ of ICRF power was coupled to the plasma in some pulses but in most of the experiments the RF power was modulated resulting in a smaller averaged $\mathrm{RF}$ power applied $\left(P_{\mathrm{RF}} \approx 2.7 \mathrm{MW}\right)$. In figure 13 , the time traces of several parameters in a typical discharge of these experiments are shown: (a) ICRF and NBI power, $(b)$ total radiated power (bolometer), $(c)$ central line-integrated density (interferometer), $(d)$ diamagnetic and equilibrium plasma energies (EFIT), $(e)$ electron (ECE) and ion (CXRS) central temperatures, $(f){ }^{3} \mathrm{He}$ concentration inferred from edge spectroscopy measurements (RTC injection network). In this discharge, a ramp of the ${ }^{3} \mathrm{He}$ concentration from $5 \%$ to $25 \%$, controlled by the real-time gas injection system, was applied $(f)$.

Because the slowing down of the ${ }^{3} \mathrm{He}$ ions is faster than that of the RF-heated $\mathrm{H}$ ions, a $4 \mathrm{~Hz} \mathrm{RF}$ power modulation (instead of a power well) was applied in several pulses to allow the assessment of the power absorption profiles and the ICRF heating efficiency. One can immediately see that although the power modulation is present in the electron temperature response throughout the discharge, it is practically absent from the $T_{\mathrm{i}}$ signals for $X\left[{ }^{3} \mathrm{He}\right]<15 \%$, indicating that under these conditions the ion absorption is very poor. For higher concentrations, the ion temperature signal starts to respond to the power modulation, reaching a similar amplitude as that observed in the electron temperature signal around 

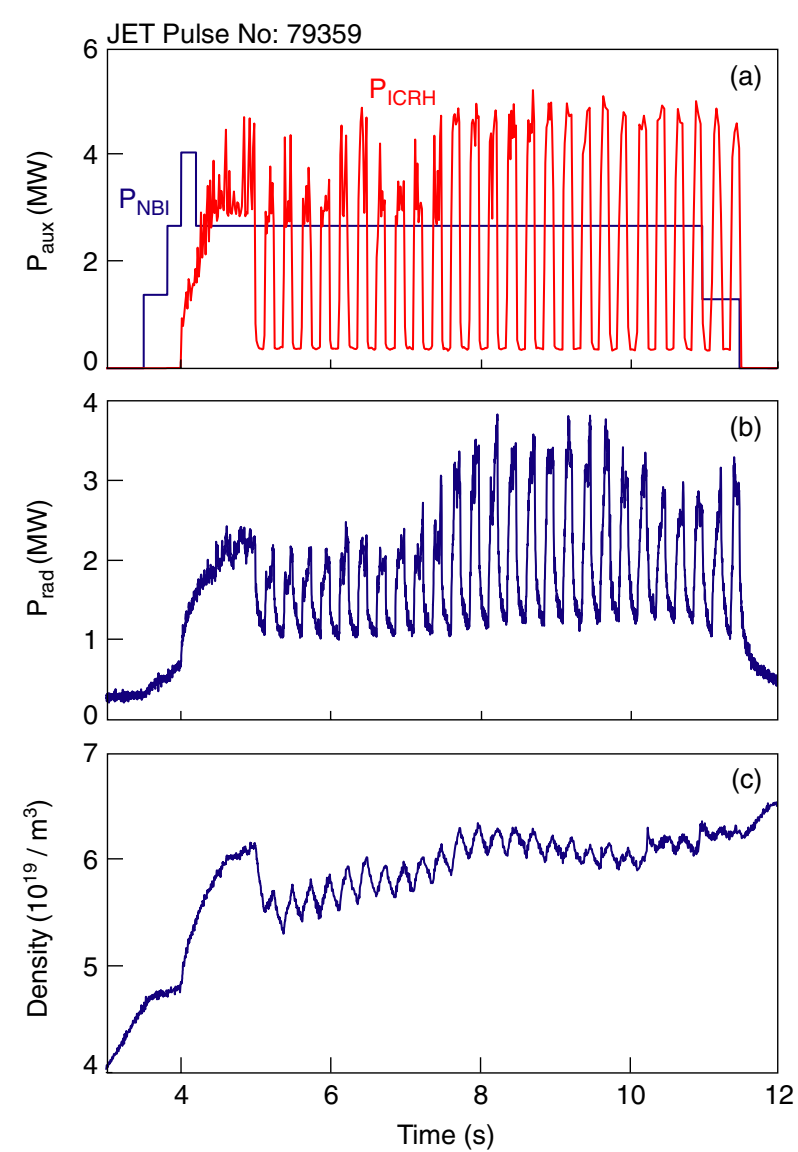
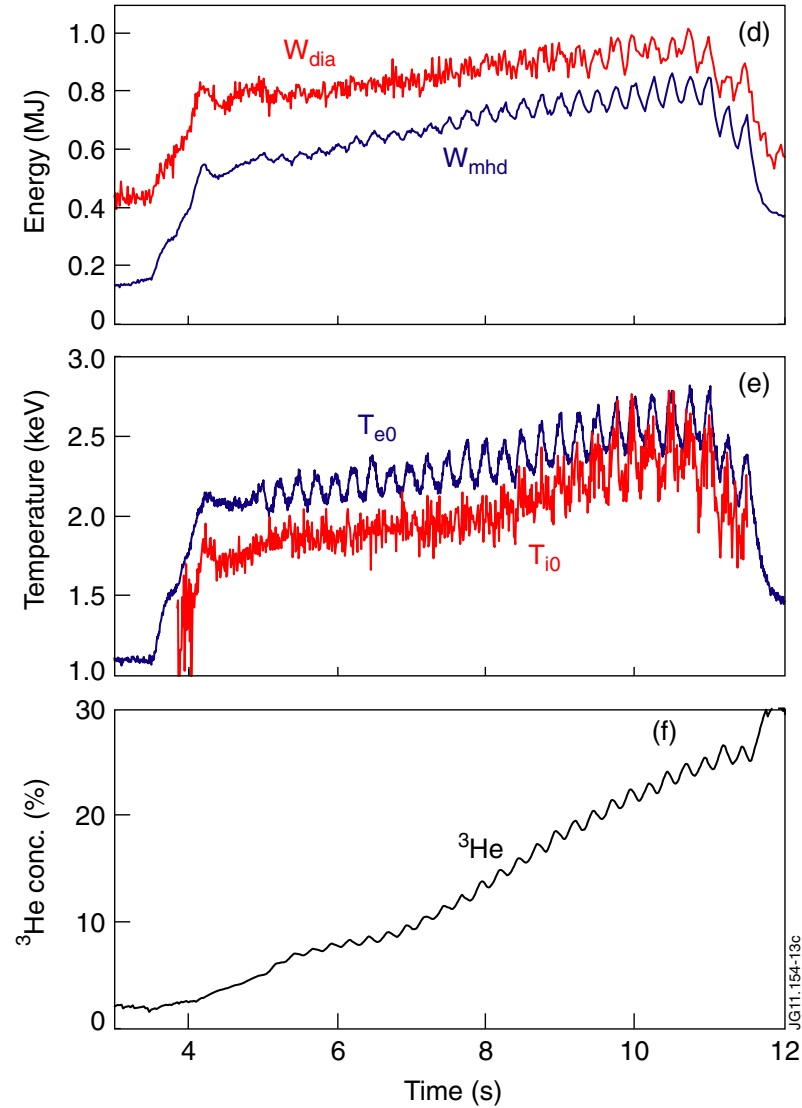

Figure 13. Typical discharge of the second harmonic ${ }^{3} \mathrm{He}$ ICRH experiments (JPN 79359): $(a)$ ICRF and NBI power, $(b)$ radiated power, $(c)$ central line-integrated density, $(d)$ diamagnetic and equilibrium plasma energies, $(e)$ electron $(\mathrm{ECE})$ and ion $(\mathrm{CXRS})$ central temperatures, $(f)^{3} \mathrm{He}$ concentration.

$X\left[{ }^{3} \mathrm{He}\right]=20-25 \%$. The energy measurements show a similar trend: the thermal energy exhibits an increasing response to the ${ }^{3} \mathrm{He}$ concentration, indicating that the overall heating efficiency is improved throughout the $X\left[{ }^{3} \mathrm{He}\right]$ ramp whereas the diamagnetic energy is practically insensitive to the RF power modulation, except for high ${ }^{3} \mathrm{He}$ concentrations. Also note the strong response of the radiated power to the RF power modulation: roughly half of the power injected into the plasma is radiated away by the impurities, indicating that the $N=2{ }^{3} \mathrm{He}$ heating scheme also exhibits a rather poor heating efficiency with enhanced plasma-wall interaction. Moreover, similarly to the fundamental $\mathrm{H}$ majority case, the diamagnetic and thermal plasma energy signals are not too far apart, indicating that in this heating scheme the ions are also not accelerated to very high energies.

\subsection{Power absorption and heating efficiency}

As mentioned before, a $4 \mathrm{~Hz}$ square wave modulation was applied to the ICRF power in several pulses to allow the assessment of the power absorption profiles and of the heating efficiency. This frequency was chosen to allow the simultaneous study of the ion and electron energy responses but is somewhat low for the determination of the actual electron power absorption, since for these time scales transport plays an important role in the energy conservation equation for the electrons [19]. In some pulses, a $25 \mathrm{MHz}$ power modulation was applied to pin down the electron absorption more accurately. In figure 14 , the power absorption profiles for ions (triangles) and electrons (circles) computed by FFT analysis of the CXRS and ECE signals, respectively, are shown for two different ${ }^{3} \mathrm{He}$ concentration 'snapshots' in pulse 79359: (a) $X\left[{ }^{3} \mathrm{He}\right] \approx 8 \%$ and $(b) X\left[{ }^{3} \mathrm{He}\right] \approx 22 \%$. The plasma density variations, which are of the order of $10 \%$ (see figure $13(c)$ ), were not considered in this analysis.

The impact of the ${ }^{3} \mathrm{He}$ concentration on the RF power absorption of both species is evident, although the effect is stronger for the ions than for the electrons. The integrated power absorption for the ions (per MW of RF power launched) is $\eta_{\mathrm{i}}=0.09$ and $\eta_{\mathrm{i}}=0.20$ for $X\left[{ }^{3} \mathrm{He}\right]=8 \%$ and $X\left[{ }^{3} \mathrm{He}\right]=$ $22 \%$, respectively, and the electron absorption varies from $\eta_{\mathrm{e}}=0.13$ to $\eta_{\mathrm{e}}=0.21$ in the two cases considered. It is important to mention once again that, because of the relatively slow RF power modulation, the integrated power absorption values computed for the electrons are influenced by transport processes and thus do not directly reflect the actual change in the electron absorption due to the change in $X\left[{ }^{3} \mathrm{He}\right]$. Similar to the figures obtained in the $\mathrm{H}$ majority heating scenario, these numbers are quite low indicating poor single-pass ICRF power absorption, particularly for low ${ }^{3} \mathrm{He}$ concentrations. The 'bump' observed in the absorption profiles of the ions at low $X\left[{ }^{3} \mathrm{He}\right]$ is thought to be related to the off-axis $N=2$ and 

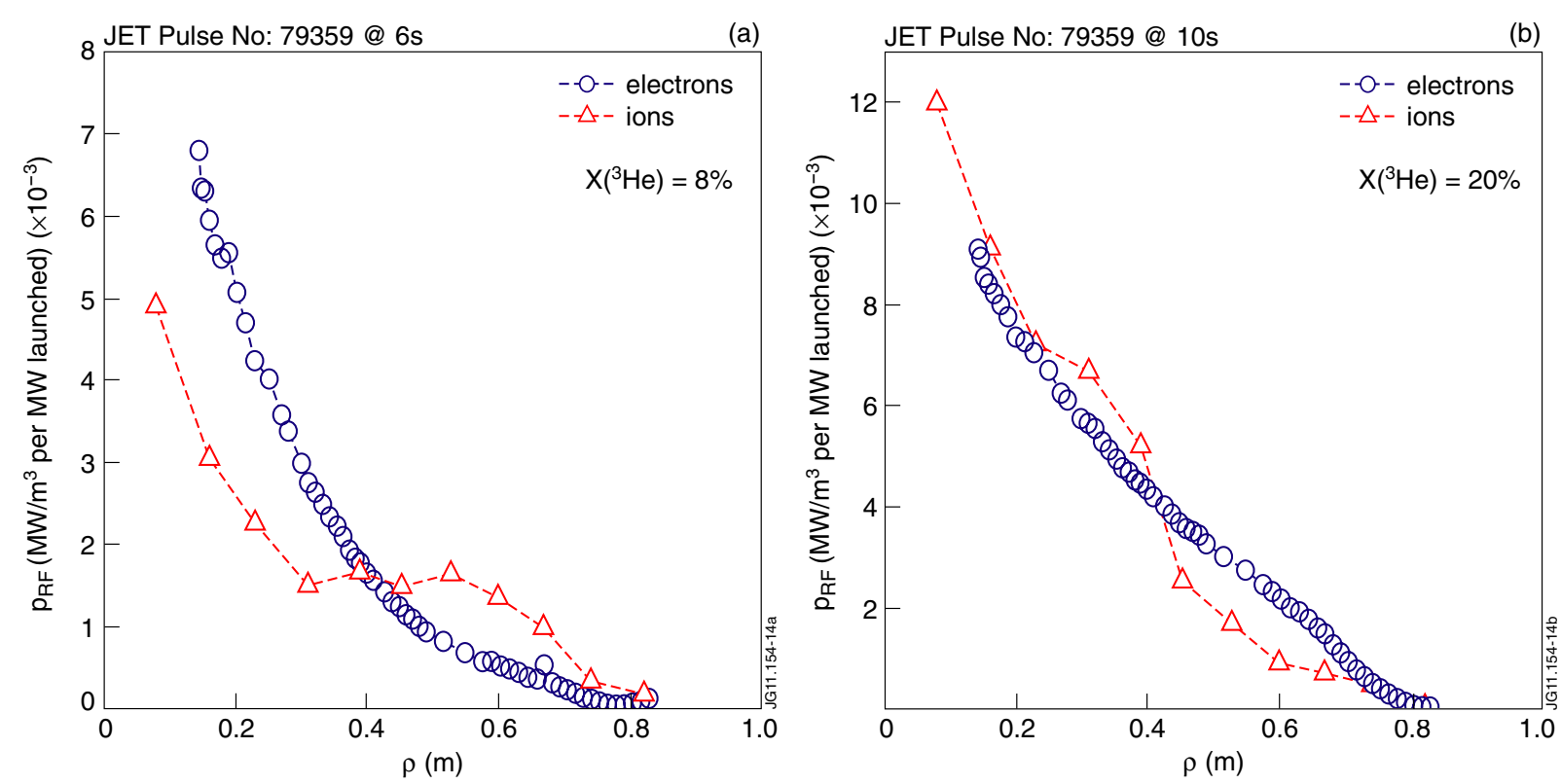

Figure 14. Power absorption profiles for the ions (triangles) and for electrons (circles) obtained by FFT analysis of the $T_{\mathrm{i}}$ and $T_{\mathrm{e}}$ responses to the $4 \mathrm{~Hz}$ RF power modulation in pulse 79359: $(a) X\left[{ }^{3} \mathrm{He}\right] \approx 8 \%(t=6 \mathrm{~s})$ and $(b) X\left[{ }^{3} \mathrm{He}\right] \approx 22 \%(t=10 \mathrm{~s})$. A time window $1 \mathrm{~s}$ was used for the FFT analysis in both cases.

$N=3$ harmonic absorption of the $\mathrm{D}$ beam ions, which is privileged under these conditions.

The enhancement of the RF heating efficiency for higher ${ }^{3} \mathrm{He}$ concentrations is depicted in more detail in figure $15(a)$, where the results obtained by FFT analysis of the plasma energy response $\left(W_{\text {mhd }}\right)$ to the RF power modulation in various discharges (circles) are plotted against the ${ }^{3} \mathrm{He}$ concentration. The latter was computed by the ${ }^{3} \mathrm{He}$ RTC network [7] based on edge spectroscopy measurements.

The fact that an improvement in the ion absorption (rather than in the electron one) is mainly responsible for the enhancement of the total heating efficiency with $X\left[{ }^{3} \mathrm{He}\right]$ is illustrated in figure $15(b)$, where the individual ion (triangles) and electron (circles) heating efficiencies obtained by analysing the central CXRS and ECE temperature signals, respectively, are plotted against the ${ }^{3} \mathrm{He}$ concentration. The data points were obtained in the same plasma discharge (79359), where a $X\left[{ }^{3} \mathrm{He}\right]$ ramp from $5 \%$ to $25 \%$ was applied (see figure 13). For the analysis, the temperature signals were multiplied by the central interferometer signal to take into account the small but non-negligible density variations due to ICRF. Furthermore, the individual heating efficiencies were normalized to give a total heating efficiency $\eta=\eta_{\mathrm{i}}+\eta_{\mathrm{e}}$ that is compatible with the values obtained by analysing the plasma energy (see the black points in figure 15(a)). Note that while the central electron response is almost insensitive to the change in ${ }^{3} \mathrm{He}$ concentration, the second harmonic ${ }^{3} \mathrm{He}$ absorption is increased by a factor of roughly 3 for $X\left[{ }^{3} \mathrm{He}\right]>20 \%$. In this regime, the ion heating efficiency alone is about $\eta_{\mathrm{i}}=25 \%$ and the ion cyclotron absorption is the dominant RF heating mechanism of the scenario. These results are in qualitative agreement with numerical predictions, which show a roughly linear dependence of the second harmonic ion cyclotron absorptivity with the concentration of the 'minority' ions.
Another clear indication of the improved heating efficiency observed in the experiments at higher ${ }^{3} \mathrm{He}$ concentrations is given in figure 16, where various quantities averaged during $0.2 \mathrm{~s}$ time intervals throughout a series of discharges are plotted against the averaged ${ }^{3} \mathrm{He}$ concentration: (a) thermal (circles) and diamagnetic (triangles) plasma energies; (b) electron (circles) and ion (triangles) central temperatures; $(c)$ central line-averaged electron density; $(d)$ total radiated power. Only the data from time intervals with similar plasma densities (as depicted in figure 16(c)), NBI power $\left(P_{\mathrm{NBI}}=1.3 \mathrm{MW}\right)$ and ICRF power $\left(P_{\mathrm{RF}} \approx 2.5 \mathrm{MW}\right)$ levels are considered. The large error bars come from the fact that most of the points correspond to time intervals in which the RF power modulation was applied.

From figure 16 it is evident that the plasma energies as well as the ion and electron temperatures achieved with the same input power increase when a larger fraction of ${ }^{3} \mathrm{He}$ is present in the plasma. Note that the ion temperatures achieved at high ${ }^{3} \mathrm{He}$ concentrations exceed the electron temperatures, in line with the fact that the ion absorption channel is dominant under these conditions. It is interesting to see that the radiated power presents a maximum around $X\left[{ }^{3} \mathrm{He}\right]=15 \%$. The decrease in the radiated power observed for $X[3 \mathrm{He}]>15 \%$ could be related to the enhancement of the heating efficiency observed at higher $X\left[{ }^{3} \mathrm{He}\right]$ (see figure 15), which could lead to a reduction in the RF power that is available for plasma-wall interactions.

\subsection{Fast particles}

Unlike for the fundamental $\mathrm{H}$ majority pulses, parasitic ion cyclotron power absorption of D ions was observed in the $N=2{ }^{3} \mathrm{He}$ heating experiments when high-energy $(E=$ $130 \mathrm{keV}$ ) neutral beams were applied to the plasma (when $80 \mathrm{keV}$ beams were used, the RF absorption of the D beam ions is much smaller). This is illustrated in figure 17 , where 

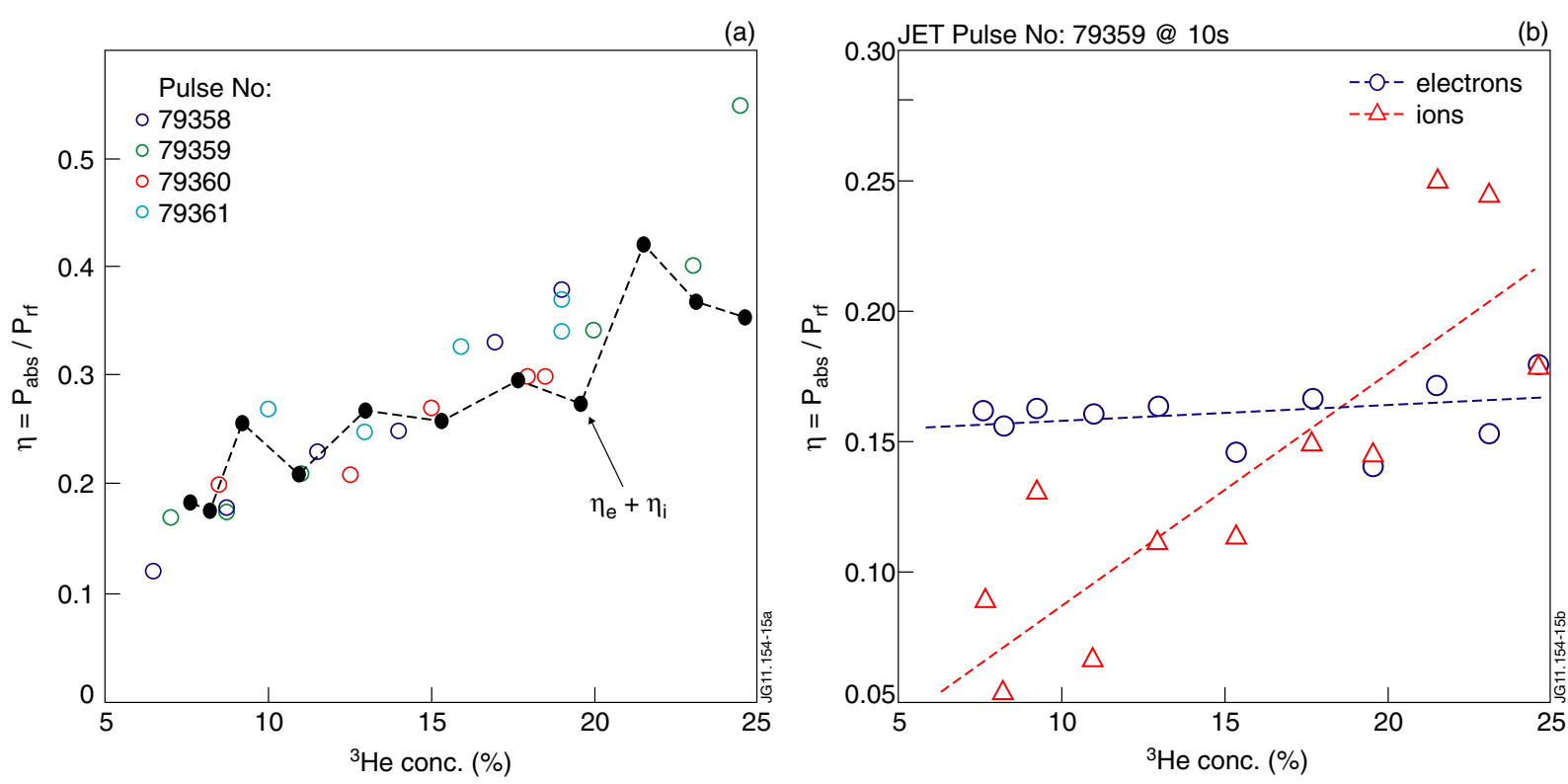

Figure 15. (a) ICRF heating efficiency as a function of the ${ }^{3} \mathrm{He}$ concentration obtained by FFT analysis of the $W_{\text {mhd }}$ signals during a $4 \mathrm{~Hz}$ RF power modulation in several plasma discharges (circles). (b) Individual heating efficiencies for electrons (circles) and ions (triangles) obtained by the FFT analysis of the central electron and ion temperatures as a function of $X\left[{ }^{3} \mathrm{He}\right]$ in pulse 79359. The black dots in $(a)$ represent the normalized heating efficiencies $\eta=\eta_{\mathrm{i}}+\eta_{\mathrm{e}}$ obtained from the data in $(b)$.

the signals of three channels of the horizontal NPA for ${ }^{3} \mathrm{He}$ (solid) and deuterium (dashed) in pulse 79632 are shown. The RF power is modulated at $4 \mathrm{~Hz}$ and the NBI power drops from 8 to $1.3 \mathrm{MW}$ (1 PINI) in the represented time interval $(a)$. The ${ }^{3} \mathrm{He}$ concentration (not shown) was maintained approximately constant at $X\left[{ }^{3} \mathrm{He}\right]=15 \%$ in this discharge.

One sees that the ${ }^{3} \mathrm{He}$ signal at $125 \mathrm{keV}$ clearly responds to the RF power modulation independently of the NBI power applied and that the signal at $E=170 \mathrm{keV}$ practically does not register any counts, indicating that the ${ }^{3} \mathrm{He}$ ions are not accelerated to very high energies under the given conditions. The $\mathrm{D}$ response in the same energy range shows the opposite trend: the lower energy channels show a limited variation to the RF power changes (being fully dominated by the counts coming from the beam 'source' ions), while the $E=170 \mathrm{keV}$ channel exhibits a clear response to the RF modulation, suggesting that RF acceleration of the $\mathrm{D}$ beam ions to energies above the beam source $(\max \sim 130 \mathrm{keV})$ takes place. In the low NBI phase, the NPA does not register any deuterons in this energy range.

A first impression of the different mechanisms concerning the ICRF power absorption of the ${ }^{3} \mathrm{He}$ and the $\mathrm{D}$ ions is illustrated in figure 18, where the average energy spectra measured by the horizontal NPA during the high-power phases (triangles) and the low-power phases (circles) of the ICRF power modulation in pulse 79362 are shown for the ${ }^{3} \mathrm{He}$ ions (a) and for the D ions $(b)$. The data represent the average values of the NPA signals in several time intervals of the high and low phases of the RF modulation taken during the $8 \mathrm{MW}$ NBI power phase $(t=5-6.5 \mathrm{~s})$. From $(a)$ we see that the number of counts is negligible when the RF power is low (the fluxes were truncated at $10^{11}$ for illustrative purposes) and that a modest ${ }^{3} \mathrm{He}$ tail with $T_{\text {eff }}=9.8 \mathrm{keV}$ is formed when $5 \mathrm{MW}$ of RF power is applied. For deuterium $(b)$, the low RF power values represent the 'beam-only' distribution (centred around $130 \mathrm{keV}$ ) with a small contribution from the collisional slowing-down of the fast $\mathrm{D}$ ions generated in the preceding high-power phases. The high RF power values (triangles) represent the averaged RF-heated D-beam distribution. However, because the slowing-down time of the $\mathrm{D}$ ions is comparable to the period of the modulation, the RFheated beam distributions have not yet achieved steady state. It is interesting to note that while the RF acceleration of the ${ }^{3} \mathrm{He}$ ions is limited to energies below $160 \mathrm{keV}$, the major part of the $\mathrm{D}$ acceleration takes place at higher energies. This is consistent with basic RF wave theory, which states that harmonic ion cyclotron power absorption is proportional to higher harmonics of the Bessel function of the first kind $J_{N}(N>1)$ and thus is more efficient for particles with supra-thermal energies such as the $\mathrm{D}$ beam ions than for the quasi-thermal ${ }^{3} \mathrm{He}$ ions [21].

Apart from the fact that the number of neutral counts detected during the RF power modulation is relatively modest, the modulation period ( $\tau=0.25 \mathrm{~s}$ ) is too short for the ion distributions to reach equilibrium after the re-application of the RF power. A better picture of the 'steady-state' ion distributions is obtained when analysing slow RF power steps. In figure 19, the time traces of three energy channels of the horizontal NPA for ${ }^{3} \mathrm{He}(b)$ and for D $(c)$ during a step in the RF power $(a)$ are shown. In this discharge, $7 \mathrm{MW}$ of NBI power using only $80 \mathrm{keV}$ PINIs was applied and the ${ }^{3} \mathrm{He}$ concentration was around $13 \%$ in the time interval represented. In figures $(d)$ and $(e)$, the respective energy spectra for ${ }^{3} \mathrm{He}$ and for $\mathrm{D}$, obtained by integrating the NPA signals in $0.8 \mathrm{~s}$ time windows during the $P_{\mathrm{RF}}=2.5 \mathrm{MW}$ (circles) and $P_{\mathrm{RF}}=5 \mathrm{MW}$ (triangles) power phases of pulse 79357, are illustrated.

From figure $19(b)$ one clearly sees that even when $5 \mathrm{MW}$ of ICRF power is applied for $1 \mathrm{~s}$, the number of counts in the ${ }^{3} \mathrm{He}$ 

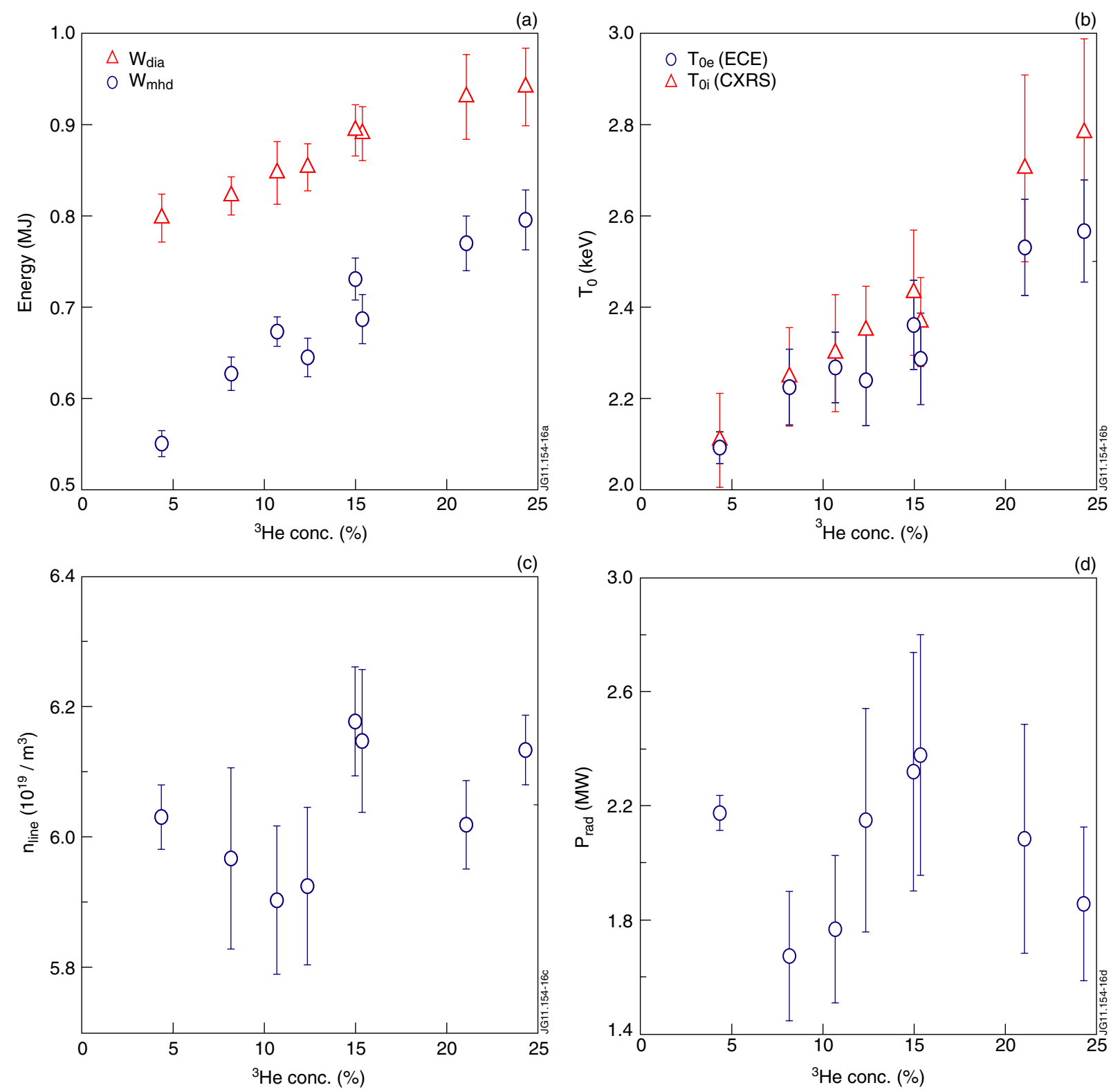

Figure 16. Several plasma parameters as a function of the ${ }^{3} \mathrm{He}$ concentration: $(a)$ thermal (circles) and diamagnetic (triangles) plasma energies; (b) electron (circles) and ion (triangles) central temperatures; $(c)$ central line-averaged electron density; $(d)$ total radiated power. The data points represent $200 \mathrm{~ms}$ time-averaged values taken from several pulses with similar densities, NBI power (1.3 MW) and ICRF power $\left(P_{\mathrm{RF}} \approx 2.5 \mathrm{MW}\right)$ levels.

channels is still small. The effective temperature of the ${ }^{3} \mathrm{He}$ distribution during the high RF power phase is $T_{\text {eff }}=9.5 \mathrm{keV}$, similar to the results obtained in the RF power modulation analysis. For the $\mathrm{D}$ ions during the low-power phase, $T_{\mathrm{eff}}=$ $27 \mathrm{keV}$, which is representative of the NBI source distribution with a small contribution of RF-heated ions accelerated above the beam source energy (mainly $80 \mathrm{keV}$ in this case). For $P_{\mathrm{RF}}=5 \mathrm{MW}$, one clearly sees the RF acceleration of the beam ions to higher energies and the effective temperature is raised to $T_{\text {eff }}=70 \mathrm{keV}$. Not only the slope of the D distribution is considerably enhanced when the RF power is larger, but the number of $\mathrm{D}$ ions with energies above $120 \mathrm{keV}$ is strongly increased.

It is important to mention at this point that although the ${ }^{3} \mathrm{He}$ ion tails measured in these experiments are considerably 'larger' than the $\mathrm{H}$ tails observed in the fundamental $\mathrm{H}$ experiments with a similar RF power, they are still very modest if compared with the RF acceleration typically measured in minority ${ }^{3} \mathrm{He}$ scenarios. On the one hand, this is related to the low heating efficiency of this heating scheme, since only a small fraction of the RF power coupled to the plasma is actually absorbed by the ${ }^{3} \mathrm{He}$ ions. On the other hand, the restricted ${ }^{3} \mathrm{He}$ $\mathrm{RF}$ acceleration observed in these experiments comes from the fact that in most of the pulses discussed here, the ${ }^{3} \mathrm{He}$ concentration was above $8 \%$, which is considerably higher than the concentrations used in typical minority ${ }^{3} \mathrm{He}-\mathrm{H}$ ICRH experiments (1-2\%), where significant ${ }^{3} \mathrm{He}$ tails were observed $[23,24]$. Furthermore, in contrast to the fundamental ion cyclotron acceleration which exhibits a relatively flat response in phase space, the second harmonic heating is usually more 
efficient at higher energies and the fact that the bulk plasma temperatures were relatively low in these pulses 'limits' this process. The parasitic ( $N=2$ and $N=3$ ) D acceleration, on the contrary, profits from the fact that most of the D ions present in these discharges come from the NBI and therefore can be more easily accelerated to high energies by harmonic ion cyclotron power absorption [21,25]. Nevertheless, the RF-induced D tails measured in these experiments are also small when compared with harmonic D ICRF heating experiments performed in the past [26], as a consequence of the low-energy/off-axis PINIs chosen for the studies reported here.

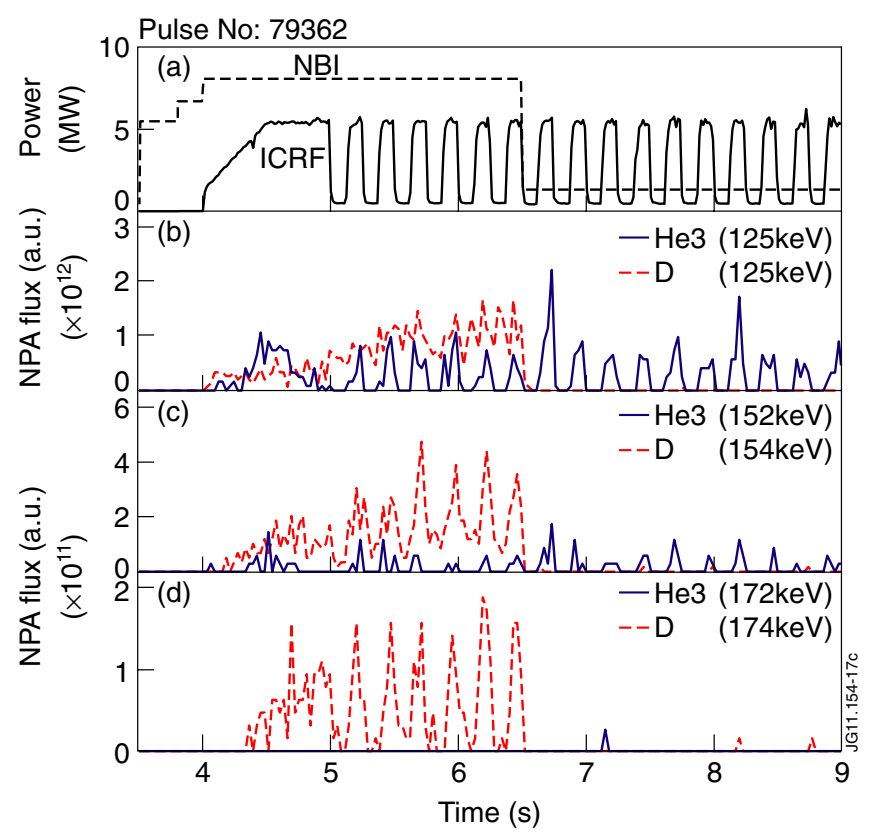

Figure 17. (a) ICRF (solid) and NBI (dashed) power signals in discharge 79362. $(b)-(d)$ Signals of three channels of the horizontal NPA for ${ }^{3} \mathrm{He}$ (solid) and deuterium (dashed) corresponding to $E \sim 125 \mathrm{keV}, E \sim 150 \mathrm{keV}$ and $E \sim 175 \mathrm{keV}$.

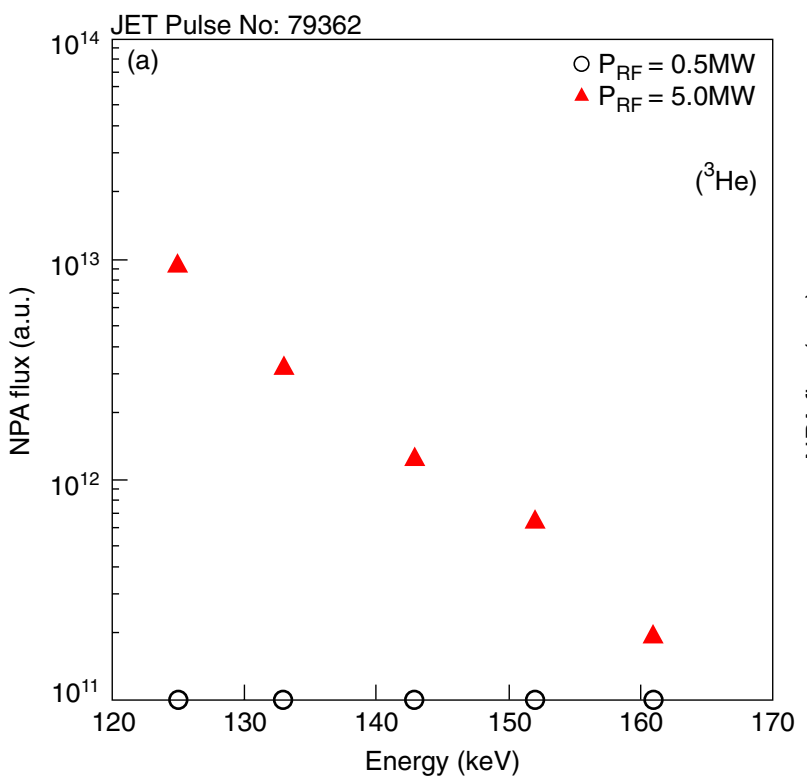

Because of the parasitic D absorption observed in these experiments, the effect of RF power absorption on the ${ }^{3} \mathrm{He}$ ion distribution is better studied when only small amounts of NBI power (or none) are applied to the plasma. Similar to the trend seen in the $\mathrm{H}$ majority experiments, the ${ }^{3} \mathrm{He}$ ion tails induced by RF also show a dependence on the density and temperature of the bulk plasma. Although a considerable sensitivity to the plasma density was also observed, the increase in the fast ion population with the plasma temperature detected in the $N=2{ }^{3} \mathrm{He}$ heating experiments is smaller than that in the fundamental $\mathrm{H}$ heating case and is thought to be mainly related to the decrease in the plasma collisionality (the $T_{\mathrm{e}}$ dependence of the heating efficiency was negligible in the $N=2{ }^{3} \mathrm{He}$ heating case). On the other hand, the NPA measurements show a clear sensitivity to the ${ }^{3} \mathrm{He}$ concentration in particular in discharges with low (or without) NBI power. Despite the fact that more efficient ion cyclotron absorption was observed at larger $X\left[{ }^{3} \mathrm{He}\right]$ (figure 15), the data indicate that the ${ }^{3} \mathrm{He}$ acceleration in the 120-160 keV energy range decreases with the ${ }^{3} \mathrm{He}$ concentration under these conditions. This effect is depicted in figure 20, where the NPA signals corresponding to ${ }^{3} \mathrm{He}$ ions with energies $E=125 \mathrm{keV}$ (figure $20(c)$ ) obtained in three different discharges with different ${ }^{3} \mathrm{He}$ concentrations (figure $20(b)$ ) are compared. The ICRF power waveform was the same in the three pulses (figure 20(a)), where the RF power was increased from 2.5 to $5.0 \mathrm{MW}$. There was no NBI power in the time interval represented and despite the different $X\left[{ }^{3} \mathrm{He}\right]$ levels, the plasma density and temperature were comparable in the high-power phase of the three discharges. In figure $20(d)$, the energy spectra for the three pulses, obtained by integrating the NPA signals from $t=11.1 \mathrm{~s}$ to $t=11.9 \mathrm{~s}$ in the $P_{\mathrm{RF}}=5 \mathrm{MW}$ phases of the discharges, are compared.

From figure 20(c) one sees that the number of counts in the $P_{\mathrm{RF}}=2.5 \mathrm{MW}$ phase is very small indicating that the number of ${ }^{3} \mathrm{He}$ ions with energies above $120 \mathrm{keV}$ is negligible. When the ICRF power is raised to $5 \mathrm{MW}$, the NPA signals start to

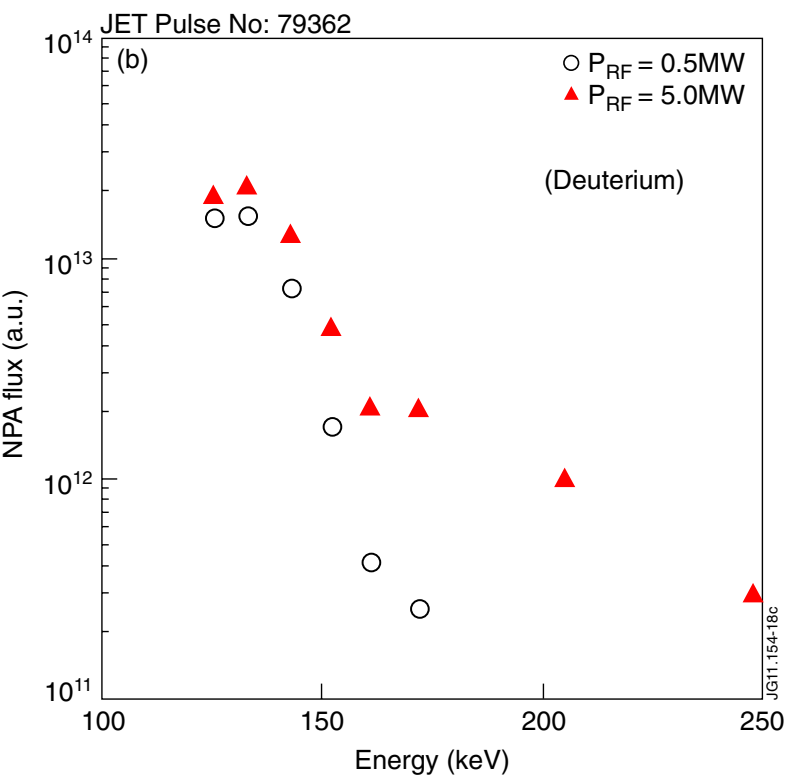

Figure 18. NPA energy spectra for $(a)^{3} \mathrm{He}$ and $(b)$ deuterium integrated during the high (triangles) and low (circles) phases of ICRF power modulation in pulse 79362 with $8 \mathrm{MW}$ of NBI power applied. 

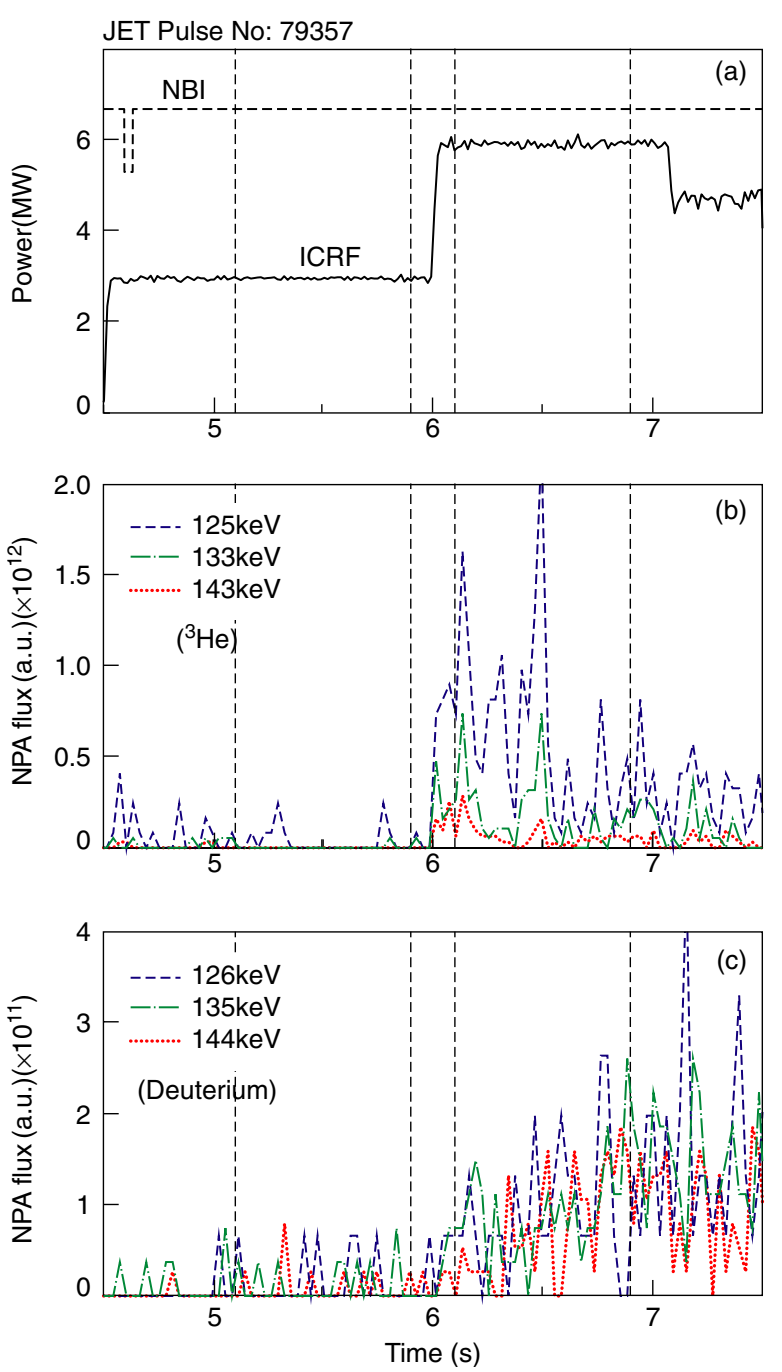
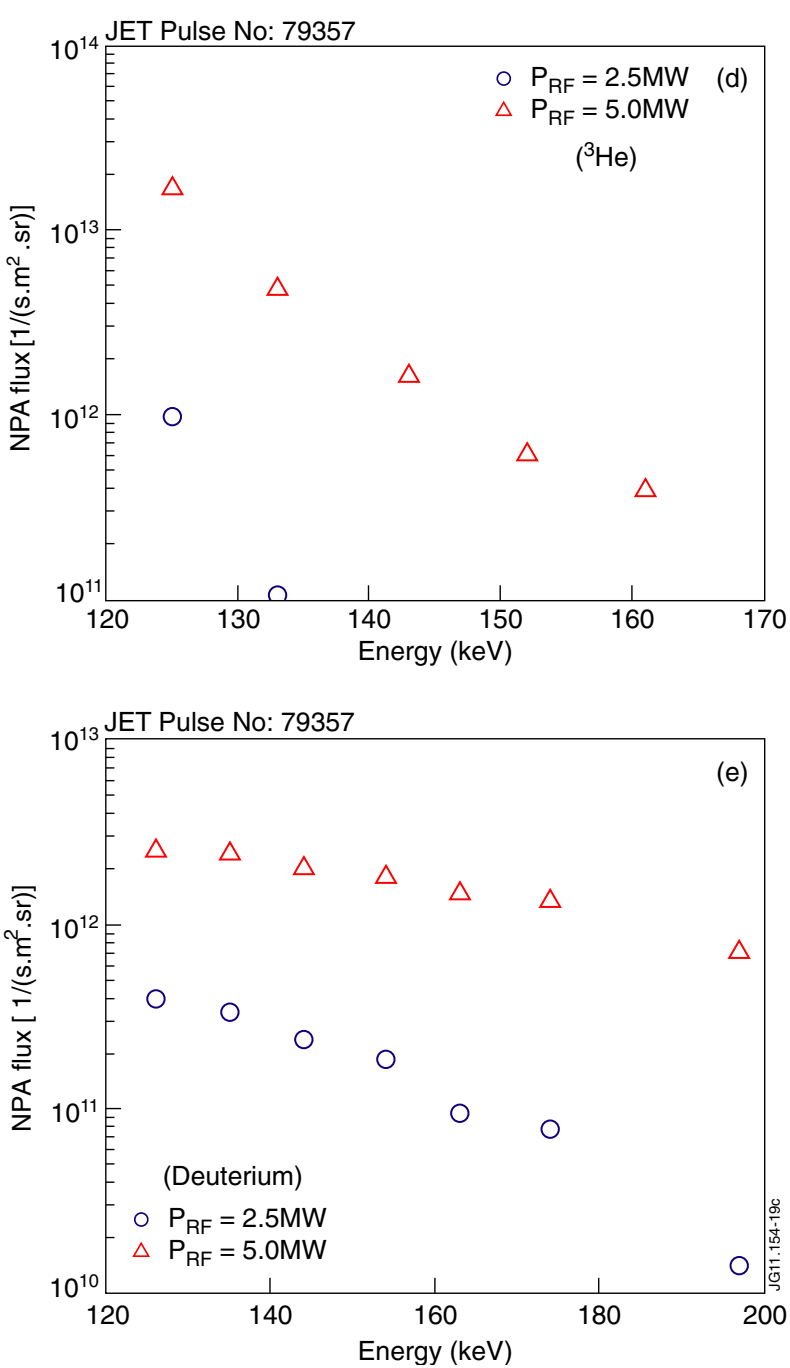

Figure 19. NPA signals for ${ }^{3} \mathrm{He}(b)$ and $\mathrm{D}(c)$ ions measured during a $2.5 \mathrm{MW}$ RF power step in pulse 79357 (a); NPA energy spectrum for ${ }^{3} \mathrm{He}(d)$ and $\mathrm{D}(e)$ when integrating the signals during $0.8 \mathrm{~s}$ in the $P_{\mathrm{RF}}=2.5 \mathrm{MW}$ phase (circles) and in the $P_{\mathrm{RF}}=5 \mathrm{MW}$ power phase. The energies of the various channels are indicated in the legends.

increase in the three cases and a clear indication of a suprathermal ion tail formation is observed (see figure $20(d)$ ). The effective temperatures are $T_{\text {eff }}=9 \mathrm{keV}, T_{\text {eff }}=11 \mathrm{keV}$ and $T_{\text {eff }}=12 \mathrm{keV}$ for $X\left[{ }^{3} \mathrm{He}\right]=17 \%, 12 \%$ and $8 \%$, respectively. Note, however, that the main effect of the ${ }^{3} \mathrm{He}$ concentration on the fast ${ }^{3} \mathrm{He}$ distribution is not on the slope of the distribution ( $\left.T_{\text {eff }}\right)$ but rather on the density of fast ions: the number of fast ${ }^{3} \mathrm{He}$ ions detected between 120 and $160 \mathrm{keV}$ is increased by about four times when the ${ }^{3} \mathrm{He}$ concentration is reduced by a factor of two. Note that the ${ }^{3} \mathrm{He}$ distribution for pulse 79362 (with similar $X\left[{ }^{3} \mathrm{He}\right]$ as pulse 79357 shown in figure 19) shows a somewhat more energetic ion tail than the results represented in figure $20(d)\left(T_{\text {eff }}=9.5 \mathrm{keV}\right)$, suggesting that the RF acceleration of the ${ }^{3} \mathrm{He}$ ions is more efficient without NBI than when $8 \mathrm{MW}$ of NBI power is applied and parasitic $\mathrm{D} /{ }^{4} \mathrm{He}$ ion cyclotron absorption occurs.

The dependence of the fast particle measurements on the ${ }^{3} \mathrm{He}$ concentration without NBI is further illustrated in figure 21, where the values of the horizontal NPA signals for $E=125 \mathrm{keV}$, averaged over $0.2 \mathrm{~ms}$ time intervals in a series of discharges with similar RF power levels $\left(P_{\mathrm{RF}} \approx 5 \mathrm{MW}\right)$ are plotted against the respective $X\left[{ }^{3} \mathrm{He}\right]$ values. These data include only values taken from time intervals with similar plasma densities $\left(n_{\text {line }} \approx 5 \times 10^{19} \mathrm{~m}^{-3}\right)$, temperatures $\left(T_{0} \approx\right.$ $3.4 \mathrm{keV}$ ) and without NBI power. When two or more PINIs are applied to the discharge together with high ICRF power, the statistical analysis did not reveal any evident trend of the NPA measurements with the ${ }^{3} \mathrm{He}$ concentration, which again suggests that the parasitic acceleration of the $\mathrm{D}$ beam ions indirectly influences the power absorbed by the ${ }^{3} \mathrm{He}$ ions.

The fact that the number of fast ${ }^{3} \mathrm{He}$ ions detected by the NPA decreases with the ${ }^{3} \mathrm{He}$ concentration notwithstanding the fact that the RF heating efficiency increases (see figure 15) suggests that for the conditions of the experiments, the enhancement of the ICRF power absorptivity is not strong enough to compensate for the increase in the number of minority ions. In other words, the net RF power absorbed per particle is smaller at higher ${ }^{3} \mathrm{He}$ concentrations despite the increase in the local power densities and thus a smaller fraction of the ${ }^{3} \mathrm{He}$ population is accelerated to higher energies. 

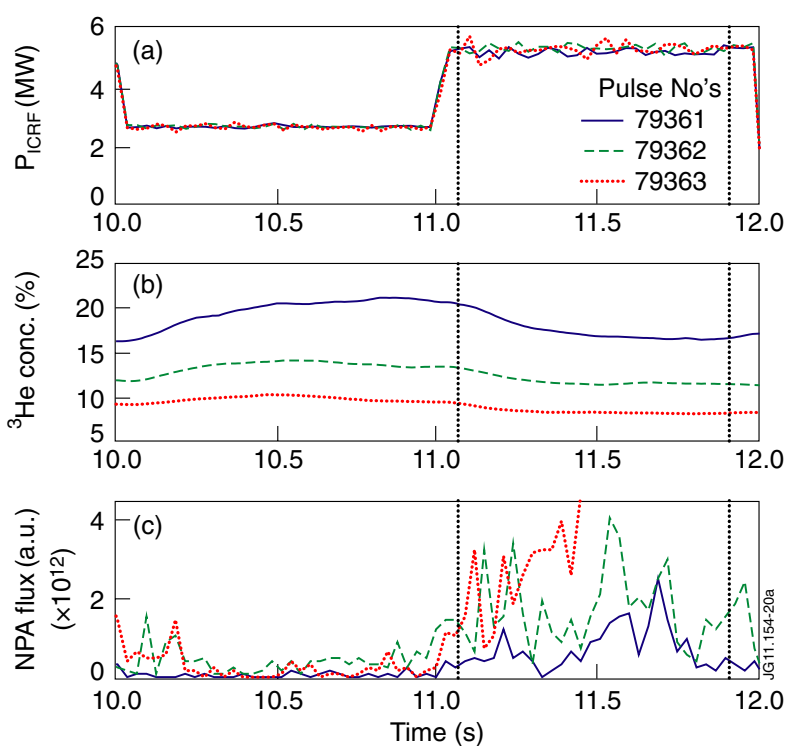

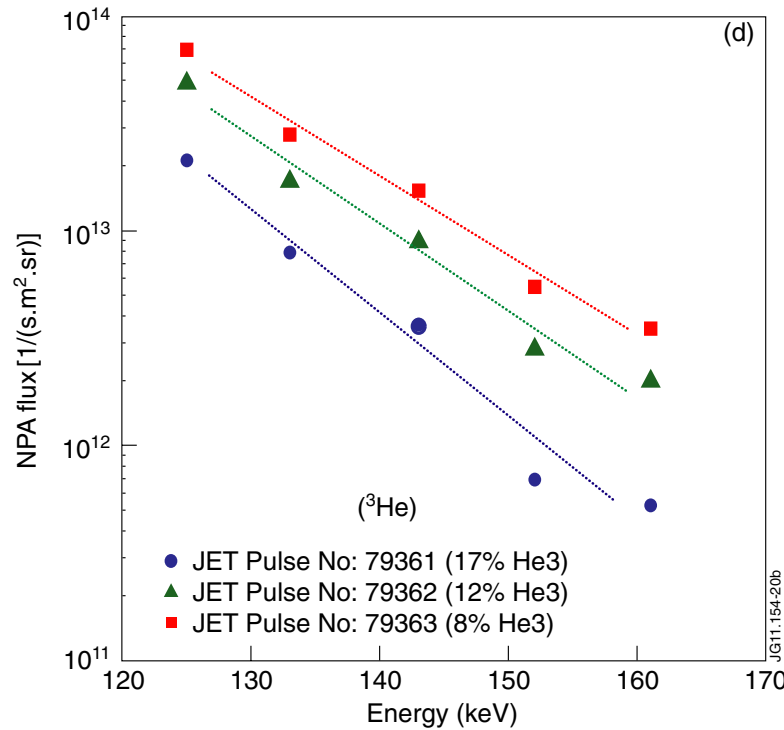

Figure 20. (a) ICRF power, $(b){ }^{3} \mathrm{He}$ concentration and $(c) E=125 \mathrm{keV}$ NPA signals for three discharges with different $X\left[{ }^{3} \mathrm{He}\right]$ values: 79361 (solid), 79362 (dashed) and 79363 (dotted); $(d)$ NPA energy spectra obtained by integrating the NPA signals during $\Delta t=11.1-11.9 \mathrm{~s}$ in the three pulses. The averaged ${ }^{3} \mathrm{He}$ concentrations in this time interval are indicated in the legend: $X\left[{ }^{3} \mathrm{He}\right]=17 \%$ (circles), $12 \%$ (triangles) and $8 \%$ (squares).

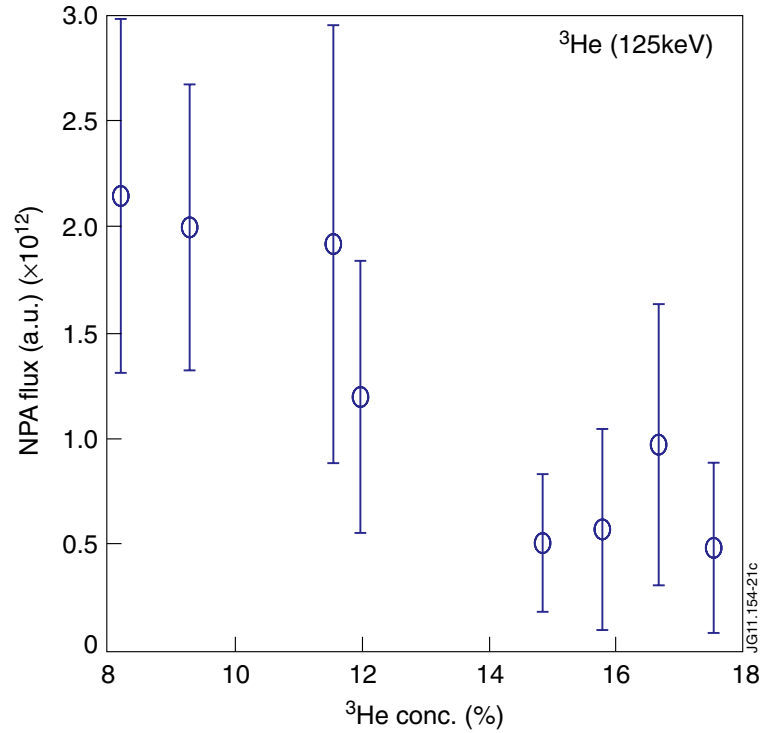

Figure 21. NPA fluxes corresponding to $E=125 \mathrm{keV}^{3} \mathrm{He}$ ions as a function of the ${ }^{3} \mathrm{He}$ concentration averaged during $0.2 \mathrm{~s}$ time intervals in several discharges. The data correspond to time intervals with the same RF power $\left(P_{\mathrm{RF}} \approx 5 \mathrm{MW}\right)$ and similar plasma densities and temperatures.

The vertical NPA (KF1), with line-of-sight close to the radial location of the $N=2{ }^{3} \mathrm{He}$ ion cyclotron resonance layer $(R=3.15 \mathrm{~m})$, was set to monitor fast ${ }^{3} \mathrm{He}$ ions with energies between 200 and $800 \mathrm{keV}$ in the experiments. An example of the results obtained with this diagnostic is shown in figure 22, where the signals of the three lowest energy channels of the vertical NPA $((b)-(d))$ registered in pulse 79363 are shown. The ICRF (solid) and NBI (dashed) power signals are represented in figure $22(a)$. The ${ }^{3} \mathrm{He}$ concentration was kept constant around $10 \%$ throughout the discharge.

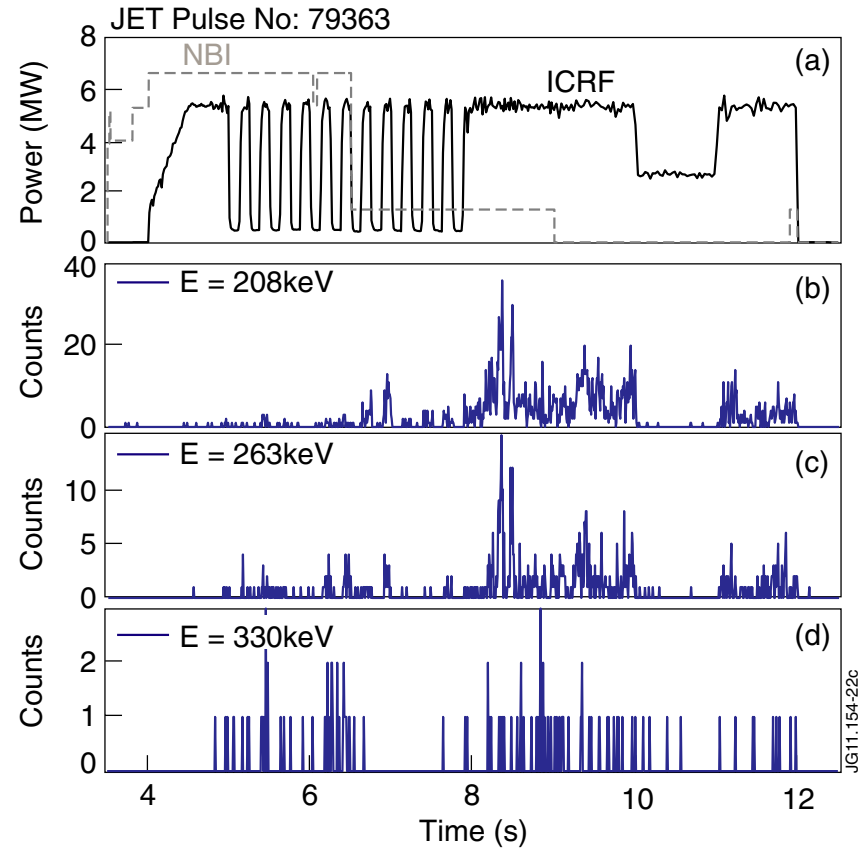

Figure 22. (a) ICRF (solid) and NBI (dashed) power waveforms in pulse 79363. $(b)-(d)$ Lowest energy channels from the vertical NPA (KF1) set to measure fast ${ }^{3} \mathrm{He}$ ions. The ion energies of the various channels are indicated in the respective legends.

Note that during the RF power modulation, a weak but visible response of fast ${ }^{3} \mathrm{He}$ ions is detected in the first two energy channels of the NPA but the number of neutral counts is very small. When the ICRF power reaches a steady state at $P_{\mathrm{RF}}=5 \mathrm{MW}$, the NPA counts become statistically relevant and RF-accelerated ${ }^{3} \mathrm{He}$ ions up to $E \sim 260 \mathrm{keV}$ are observed. The channels corresponding to fast ions with $E \geqslant$ $330 \mathrm{keV}$ practically do not register any counts independently 


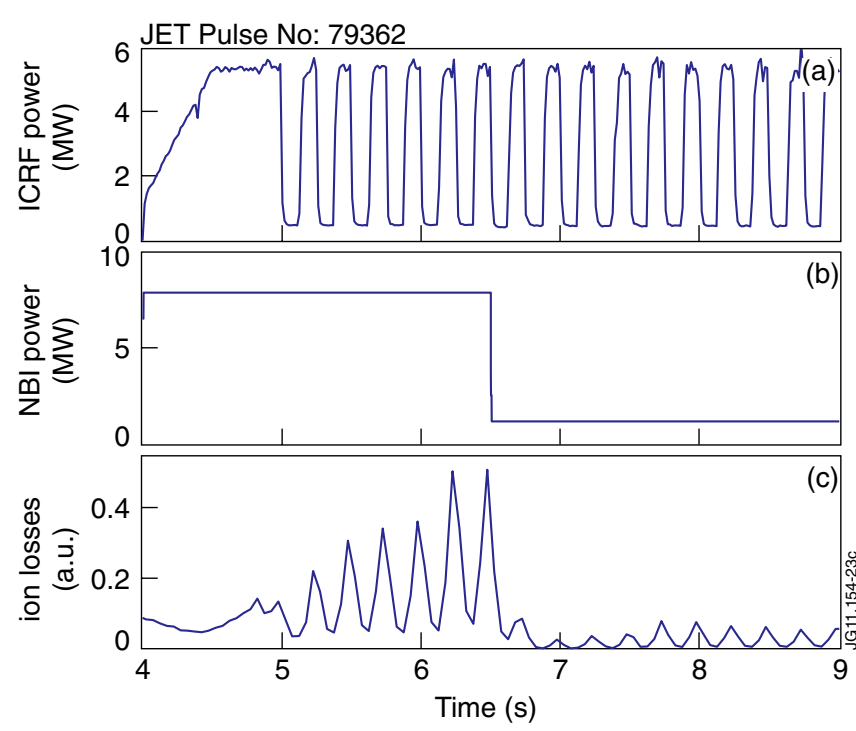

Figure 23. (a) RF power, (b) NBI power and (c) total fast ion losses detected by the scintillator probe in pulse 79362 .

of the ICRF power applied (figure 22(d)). Unfortunately, this diagnostic was not set to measure fast $\mathrm{D}$ ions in this session and thus the parasitic acceleration of the beam injected $\mathrm{D}$ ions to higher energies observed in some of the other diagnostics could not be confirmed.

The studies of the fast ${ }^{3} \mathrm{He}$ and $\mathrm{D}$ populations driven by ICRF in the reported experiments were further supported by the measurement of fast ion losses performed by a scintillator probe (KA3) located in the LFS of the machine about $0.5 \mathrm{~m}$ below the mid-plane [12]. This probe is capable of measuring fast ions that escape the plasma with Larmor radii varying from $\rho_{\mathrm{L}}=3 \mathrm{~cm}$ to $\rho_{\mathrm{L}}=13 \mathrm{~cm}$ and pitch angles between $40^{\circ}$ and $80^{\circ}$. The time evolution of the total ion losses detected by the scintillator probe (integrated over $\rho_{\mathrm{L}}$ and pitch angle) in pulse 79362 is shown in figure 23(c), together with the ICRF power $(a)$ and NBI power $(b)$ waveforms applied in this discharge.

Note that the ion losses are much stronger during the high NBI power phase $(t<6.5 \mathrm{~s})$ than when the NBI is reduced to $1.3 \mathrm{MW}$, indicating that the main losses detected by the scintillator probe come from RF-accelerated beam ions rather than from fast ${ }^{3} \mathrm{He}$ ions (the modest contribution of the fast ${ }^{3} \mathrm{He}$ ions to the measured losses can be seen during the lowpower NBI phase). This observation is in line with the estimate of the fast ion distributions inferred from NPA measurements (figure 19), which indicate that the RF acceleration of the $\mathrm{D}$ beam ions to high energies is more efficient than the ${ }^{3} \mathrm{He}$ acceleration particularly when higher level of NBI power is applied to the discharge. Gamma-ray measurements suggest, however, that the D ions are not accelerated to energies above $500 \mathrm{keV}$ in any of the discharges. A more detailed discussion of the fast ions observed in these experiments is presented in another paper of this edition [27].

\subsection{Global performance and confinement}

Similar to the performance study done for the fundamental $\mathrm{H}$ majority heating experiments (section 2.4), a statistical analysis based on time-averaged values of various signals taken in several pulses throughout the session was used to assess the dependence of the key plasma parameters on the ICRF power applied. Eleven time intervals of $\Delta t=0.2 \mathrm{~s}$ in nine different discharges were used for these studies and the data were subdivided according to NBI power, plasma density and ${ }^{3} \mathrm{He}$ concentration values. In figure 24 , the averaged values of some plasma parameters are plotted against the average ICRF power coupled to the plasma: $(a)$ central electron temperature, (b) central ion temperature, $(c)$ total radiated power and $(d)$ energy confinement time. The circles represent intervals with $P_{\mathrm{NBI}}=1.3 \mathrm{MW}(1 \mathrm{PINI})$ while the triangles correspond to intervals with $P_{\mathrm{NBI}}=6.5 \mathrm{MW}$ (5 PINIs). The values at $P_{\text {ICRH }}=0$ represent the averaged quantities before the ICRF power was applied. The central line-averaged plasma densities (not shown) were about $n_{\text {line }}=(5.5 \pm 0.5) \times 10^{19} \mathrm{~m}^{-3}$ and $n_{\text {line }}=(6.1 \pm 0.2) \times 10^{19} \mathrm{~m}^{-3}$ in the time intervals with 1 PINI and with 5 PINIs, respectively, and did not show any systematic increase with the ICRF power. The ${ }^{3} \mathrm{He}$ concentration varies between $10 \%$ and $20 \%$ in the chosen data set. The error bars indicate the standard deviation of the average values in each of the time intervals considered. The points with larger error bars correspond to intervals where the $4 \mathrm{~Hz} \mathrm{RF}$ power modulation was applied.

Both the electron and ion temperatures measured in the two NBI power levels show a roughly linear increase with the ICRF power applied. The slopes are similar for the low and high NBI power cases with $\Delta T_{\mathrm{e}} / \Delta P_{\mathrm{RF}}=0.18$ and $\Delta T_{\mathrm{i}} / \Delta P_{\mathrm{RF}}=0.15 \mathrm{keV} \mathrm{MW}^{-1}$ for the electrons and for the ions, respectively (the point with $P_{\mathrm{ICRF}}=0$ was discarded in all cases). The uncertainties in these coefficients are large, since the linear fit is very sensitive to the data points at high RF power levels, which are rather scarce, especially for the low $P_{\mathrm{NBI}}$ data set. The fact that the slopes obtained for low and high NBI power are similar indicates that the overall heating efficiency of this scenario is not very sensitive to the plasma temperature. Even if these slopes are somewhat higher than those obtained in similar conditions in the fundamental $\mathrm{H}$ majority ICRH experiments, the values are still much smaller than the values typically reached in standard $\mathrm{H}$ minority scenarios (which can reach values of $1 \mathrm{keV} \mathrm{MW}^{-1}$ ). Once again, this low plasma performance is due to a combination of the relatively low ICRF heating efficiency of the $N=2$ ${ }^{3} \mathrm{He}$ scenario at the sampled $X\left[{ }^{3} \mathrm{He}\right]$ values, high radiation losses due to enhanced RF-induced plasma-wall interaction and poor confinement of the hydrogen plasma at $2.65 \mathrm{~T}$. From figure 24(c) we see that for the low NBI power case, the radiated power is augmented by $\sim 2 \mathrm{MW}$ when the ICRF power is increased from 1 to $6 \mathrm{MW}$, indicating that roughly half of the RF power applied is lost by radiation in the ICRFheated discharges. The energy confinement time is degraded by a factor of two in the same power interval. The energy confinement of the plasma is much less sensitive to the RF power when higher NBI power levels are applied. The fact that the $\tau_{E}$ values are only about $0.1 \mathrm{~s}$ when $6.5 \mathrm{MW}$ of NBI power is applied (even at low $P_{\mathrm{ICRF}}$ ) depicts the low confinement regime of these plasmas.

Note that the $\Delta T / \Delta P_{\mathrm{RF}}$ slopes obtained in the $N=2$ ${ }^{3} \mathrm{He}$ ICRH experiments were somewhat higher than those 

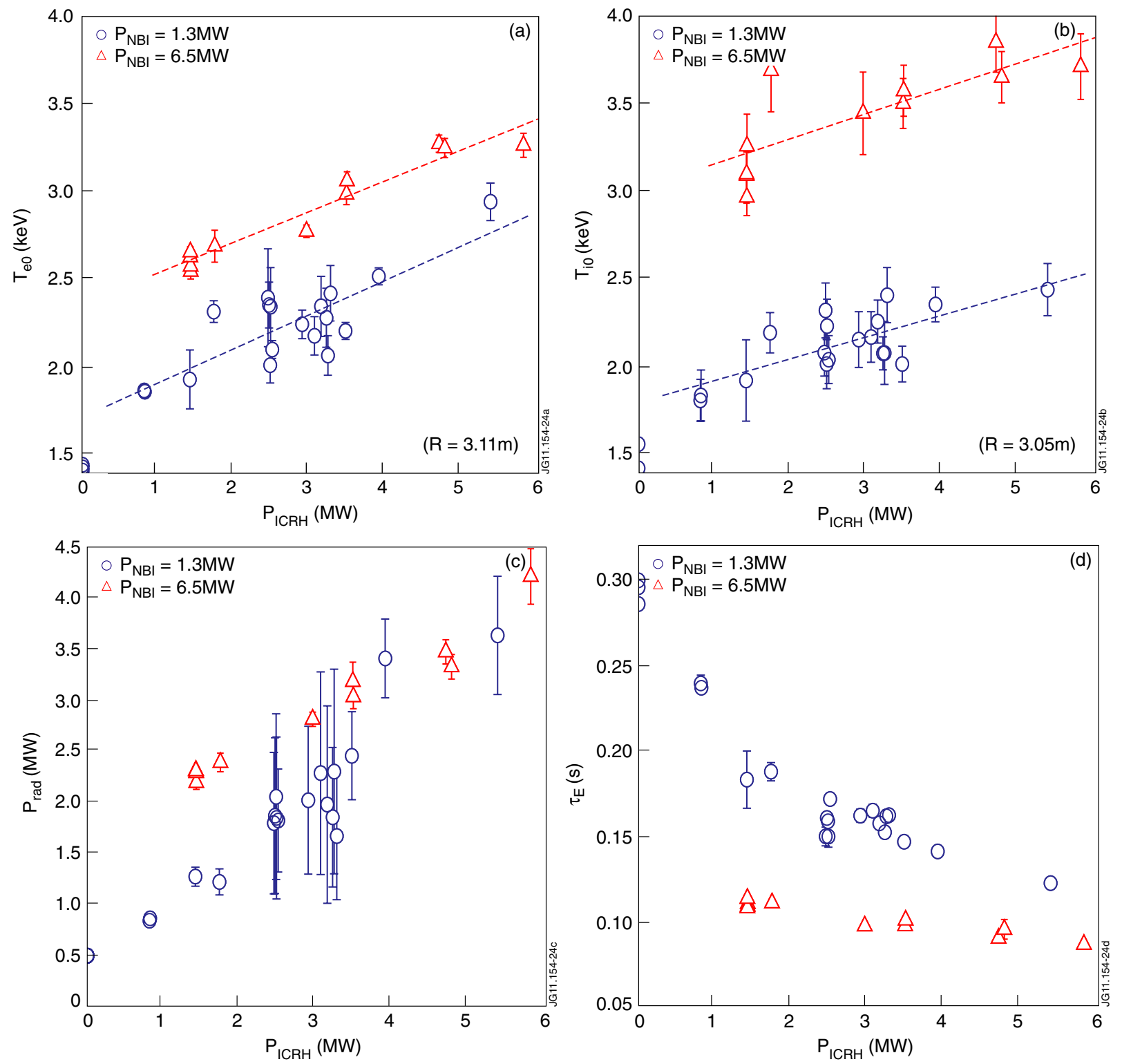

Figure 24. Averaged values of several signals as a function of the ICRF power during $0.2 \mathrm{~s}$ time intervals sampled in various discharges with $1.3 \mathrm{MW}$ (circles) and 6.5 MW (triangles) NBI power: $(a)$ central electron temperature (ECE at $R=3.11 \mathrm{~m}$ ); $(b)$ central ion temperature (CXRS at $R=3.05 \mathrm{~m}$ ); (c) total radiated power (bolometer); $(d)$ energy confinement time $\tau_{E}$.

obtained in the fundamental $\mathrm{H}$ majority ICRH studies, despite their similar ICRF heating efficiencies $(\eta=0.3-$ 0.4). This is a consequence of several factors: (i) the ion cyclotron heating mechanism is different for the two cases leading to different power absorption profiles and to distinct RF-heated ion distributions and fast ion losses; (ii) the transport processes in a 'pure' hydrogen plasma and in a $X\left[{ }^{3} \mathrm{He}\right]=15-20 \%$ diluted $\mathrm{H}$ plasma (higher $Z_{\text {eff }}$ ) are also dissimilar, eventually leading to different species densities and temperature profiles; (iii) the two heating scenarios use different RF frequencies leading to distinct RF-induced plasma-wall interactions and thus different radiation losses. A more detailed comparison between the fundamental $\mathrm{H}$ majority and the second harmonic ${ }^{3} \mathrm{He}$ heating schemes described in the previous sections will be presented subsequently.

\section{Impurity generation and performance comparison}

Apart from the different RF settings and the somewhat different plasma compositions, the $N=1 \mathrm{H}$ and the $N=2$ ${ }^{3} \mathrm{He}$ ICRF heating experiments were performed under similar plasma conditions, both having the same plasma geometry and approximate equilibrium parameters. Nevertheless, clear differences in the impurity content of the plasma during ICRF heating (and distinct transport properties) were observed in the two scenarios. In figure 25, several impurity related quantities obtained in the $N=1 \mathrm{H}$ (circles) and in the $N=2$ ${ }^{3} \mathrm{He}$ discharges (triangles) plotted as a function of the ICRF power are compared: (a) total radiated power (bolometer), (b) effective charge (visible spectroscopy), (c) beryllium line intensity (visible spectroscopy) and $(d)$ nickel concentration 

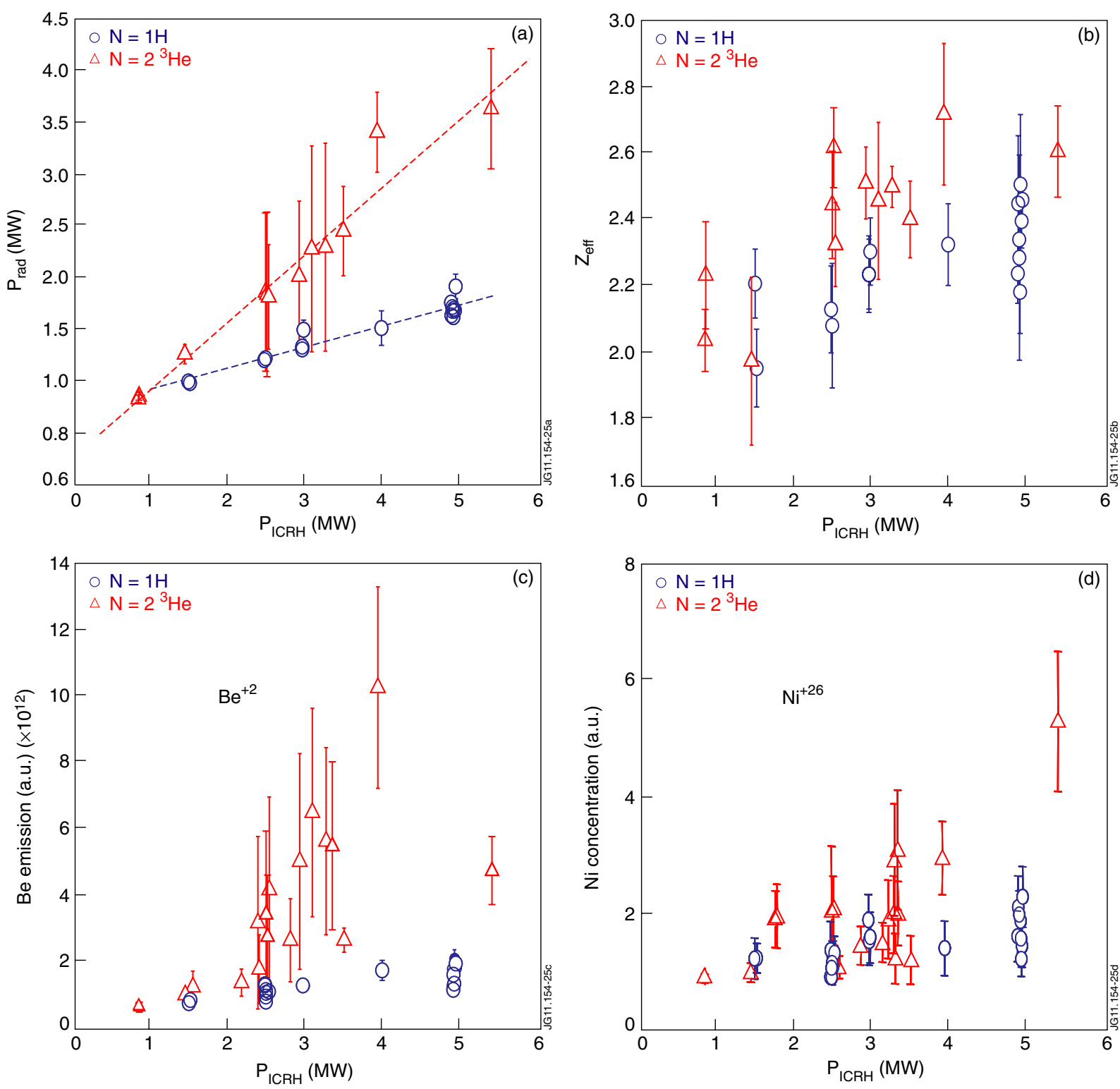

Figure 25. Comparison of impurity generation in the $N=1 \mathrm{H}$ (circles) and in the $N=2{ }^{3} \mathrm{He}$ (triangles) heating discharges: (a) total radiated power (bolometer), (b) effective charge (visible spectroscopy), (c) beryllium line intensity (visible spectroscopy) and $(d)$ nickel concentration (VUV spectroscopy).

(VUV spectroscopy). The data points of the two sessions correspond to $0.2 \mathrm{~s}$ time averages of the various signals and were taken during instants where the density, NBI power $(1.3 \mathrm{MW})$ and ohmic power $(\sim 0.7 \mathrm{MW})$ were similar. The large error bars present in most of the ${ }^{3} \mathrm{He}$ session data points are due to the fact that many time intervals were taken during the RF power modulation phase of the discharges.

The slopes represented in figure 25( $a$ ) (obtained by linear fit of the data points) are $\alpha=0.25 \mathrm{MW}$ per MW ICRF power for the $N=1 \mathrm{H}$ data and $\alpha=0.61 \mathrm{MW}$ per MW ICRF power for the $N=2{ }^{3} \mathrm{He}$ session data. The radiation losses are clearly much more significant in the $N=2{ }^{3} \mathrm{He}$ case than in the $N=1 \mathrm{H}$ heating pulses for the same input power and are responsible for roughly $50 \%$ of the total losses at high $\mathrm{RF}$ power in these discharges. The higher radiation losses in the ${ }^{3} \mathrm{He}$ heating discharges are in line with the higher $Z_{\text {eff }}$ inferred from visible spectroscopy, as shown in figure $25(b)$.
The larger $Z_{\text {eff }}$ values observed in these experiments are not only due to the presence of ${ }^{3} \mathrm{He}$ ions in the plasma, but also due to the enhanced RF-induced impurity content observed in the $N=2{ }^{3} \mathrm{He}$ heating pulses, as shown in figures $25(c)$ and (d) for beryllium and nickel, respectively. The large spread of the data points in the $\mathrm{H}$ heating experiments at high ICRH powers are due to the different levels of ${ }^{4} \mathrm{He}$ present in these discharges, as discussed in section 2.5.

From figure 25 it is clear that the higher metallic impurity content observed in the ${ }^{3} \mathrm{He}$ heating experiments is related to enhanced plasma-wall interaction due to the application of ICRF power, mainly caused by non-resonant acceleration of charged particles in the scrape-off layer (sheath rectification effects) and by direct sputtering of the wall material by fast ions lost from the plasma. The main source of beryllium is the Faraday screen of the ICRF antenna array while the main nickel sources are believed to be the screws that fix the 

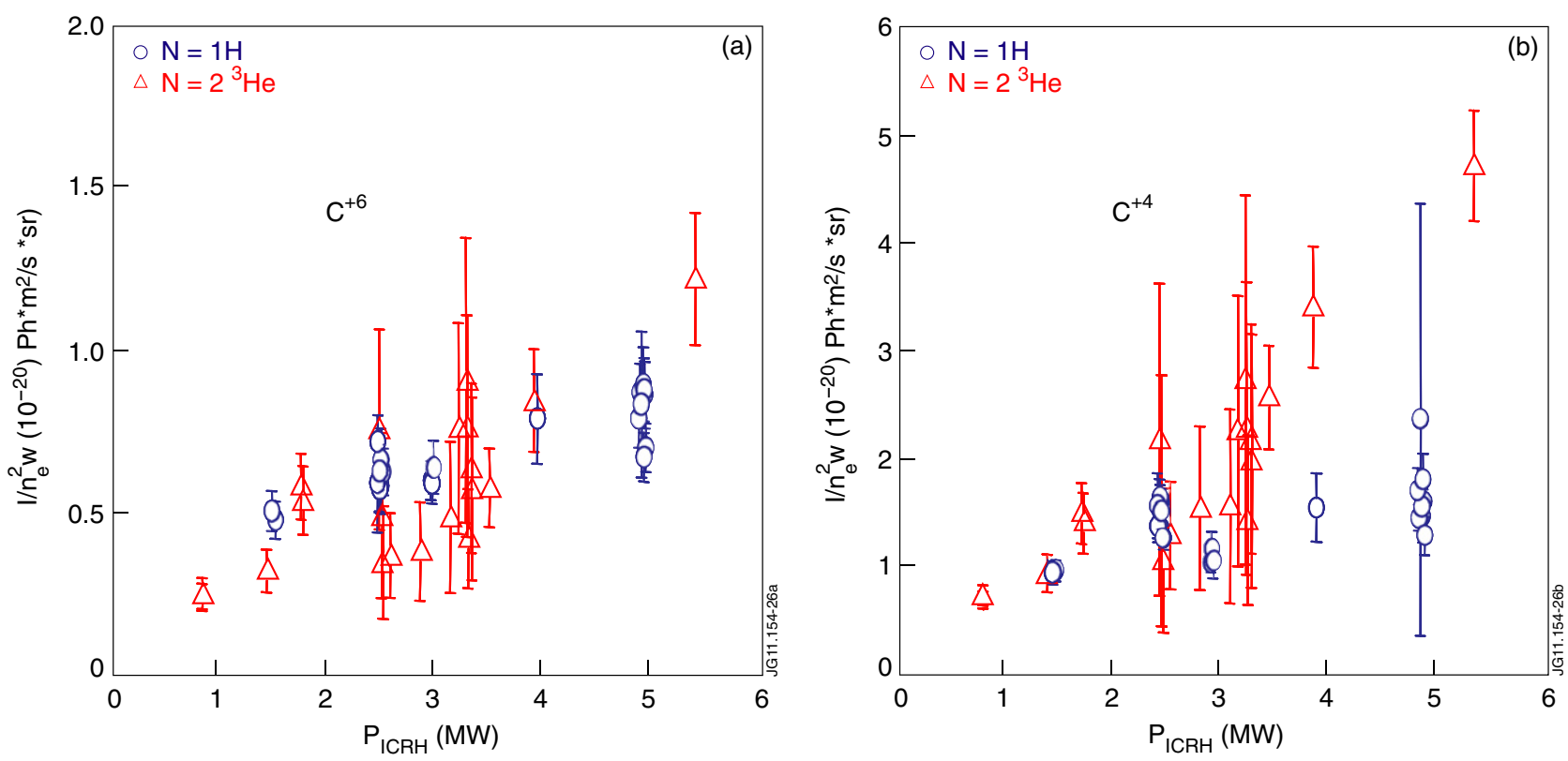

Figure 26. $\mathrm{C}^{6+}(a)$ and $\mathrm{C}^{4+}(b)$ concentrations measured by VUV spectroscopy as a function of the ICRF power in the $N=1 \mathrm{H}$ (circles) and in the $N=2{ }^{3} \mathrm{He}$ (triangles) heating experiments.

Faraday screens to the antenna boxes. However, sputtering of $\mathrm{Be}$ and $\mathrm{Ni}$ deposited in other regions of the vessel wall (during past experiments) also probably takes place but a quantitative analysis of this contribution to the total Ni content in the plasma was never done. A more detailed study of the impurity influx generated during ICRF heating including nickel and various other metallic impurities is given in another paper of this special edition [28].

In figure 26, the $\mathrm{C}^{6+}(a)$ and $\mathrm{C}^{4+}(b)$ concentrations inferred from VUV spectroscopy (KT7) averaged in several time intervals throughout the $N=1 \mathrm{H}$ (circles) and the $N=2$ ${ }^{3} \mathrm{He}$ (triangles) heating experiments are plotted as a function of the RF power. It is interesting to note that although the $\mathrm{C}^{6+}$ content is similar in the two cases, the $\mathrm{C}^{4+}$ concentration exhibits a much stronger increase with ICRF power in the $N=2{ }^{3} \mathrm{He}$ heating case, suggesting that most of the radiation losses in these discharges come from the outer region of the plasma rather than from the plasma core. 2D bolometric tomography [29] confirmed this hypothesis and revealed that a significant part of the radiation observed in the ${ }^{3} \mathrm{He}$ heating discharges comes from the plasma periphery and from the divertor region.

The different RF-induced impurity behaviour observed in the two cases has an impact on the overall equilibrium of the two heating schemes. If for the same ICRF input power (and constant NBI and ohmic power) the radiation losses are higher in a set of plasma discharges than in others with a similar magnetic configuration, then the remaining power losses $\left(P_{\mathrm{TOT}}-P_{\mathrm{RAD}}\right)$, imposing the energy balance of the plasma, must be lower and vice versa. This is depicted in figure $27(a)$, where the power differences $P_{\mathrm{TOT}}-P_{\mathrm{RAD}}$ for the same time intervals represented in figure 25 are shown for the $N=1 \mathrm{H}$ (circles) and $N=2{ }^{3} \mathrm{He}$ (triangles) heating cases. The points forming the dotted line represent the total power applied during each time interval, $P_{\text {TOT }}=$
$P_{\mathrm{ohm}}+P_{\mathrm{NBI}}+P_{\mathrm{ICRH}}$, with $P_{\mathrm{NBI}}=1.3 \mathrm{MW}$ and $P_{\mathrm{ohm}}=0.7 \mathrm{MW}$ practically constant in the data set. If one assumes that these data correspond to time intervals in which the discharges were in 'thermal equilibrium' $(\mathrm{d} W / \mathrm{d} t=0)$, then the difference $P_{\text {loss }}=P_{\mathrm{TOT}}-P_{\mathrm{RAD}}$ represents all the other (non-radiation related) losses in the plasma, which are mainly related to energy and particle transport processes (ripple losses, fast ion losses and radiation losses not captured by the bolometer are second-order corrections to the overall losses under the given experimental conditions). The small standard deviation of the plasma energy signals (not shown) suggests that the plasma was indeed in approximate thermal equilibrium in most of the time intervals represented.

The fact that the energy confinement time values are very similar in the two experiments, as shown in figure $27(b)$ for the same time intervals, indicates that the total losses and that the global confinement are similar in the two heating scenarios. The slopes indicated in figure 27( $a$ ) are $\alpha=0.75 \mathrm{MW}$ (for $N=1 \mathrm{H}$ ) and $\alpha=0.35 \mathrm{MW}$ (for $N=2{ }^{3} \mathrm{He}$ ) per MW of ICRF power applied and clearly reflect the fact that the transport losses are the main players in the equilibrium of the $N=1 \mathrm{H}$ discharges, being responsible for about $70 \%$ of the total losses at $P_{\mathrm{ICRH}}=5 \mathrm{MW}$ in this case. Therefore, despite the similar RF heating efficiencies and global confinement properties of the two scenarios, their corresponding equilibrium conditions are different: in the $N=1 \mathrm{H}$ heating discharges, most of the applied power is lost by transport and the radiation losses only contribute to a small fraction to the overall energy balance; oppositely, in the $N=2{ }^{3} \mathrm{He}$ discharges, both radiation and transport losses have similar weights in establishing the plasma equilibrium. Moreover, in the discharges with high radiation losses, most of the power is lost from the outer surfaces of the plasma while in the transport-dominated plasmas, one of the main loss mechanisms is the energy diffusion from the plasma core to the periphery. Consequently, one could expect 

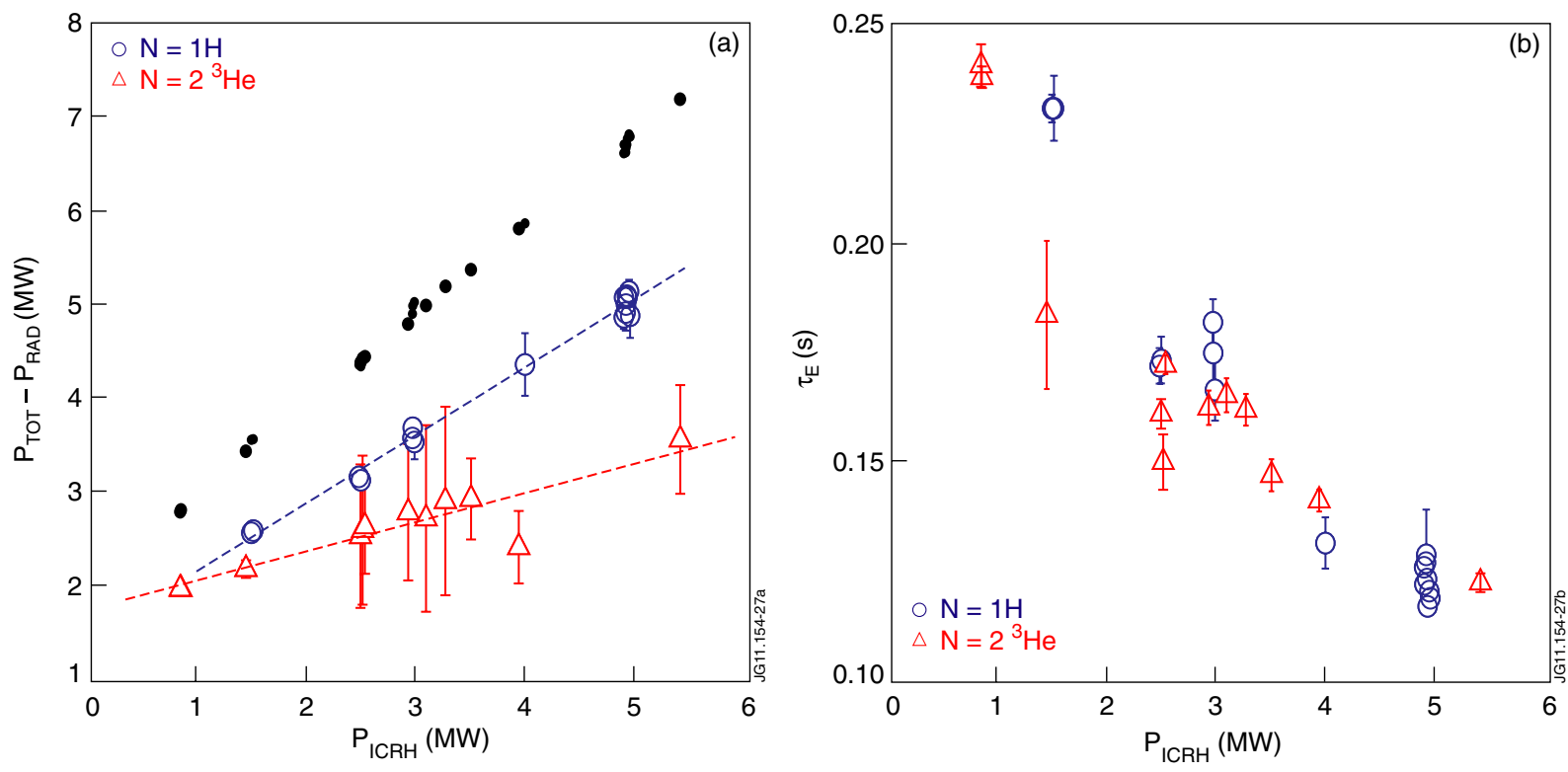

Figure 27. (a) Non-radiative power losses $P_{\mathrm{TOT}}-P_{\mathrm{RAD}}$ for the $N=1 \mathrm{H}$ (circles) and $N=2{ }^{3} \mathrm{He}$ (triangles) heating cases as a function of the applied ICRF power (small points represent the total power applied $P_{\mathrm{TOT}}$ ). (b) Averaged energy confinement times for the same time intervals.
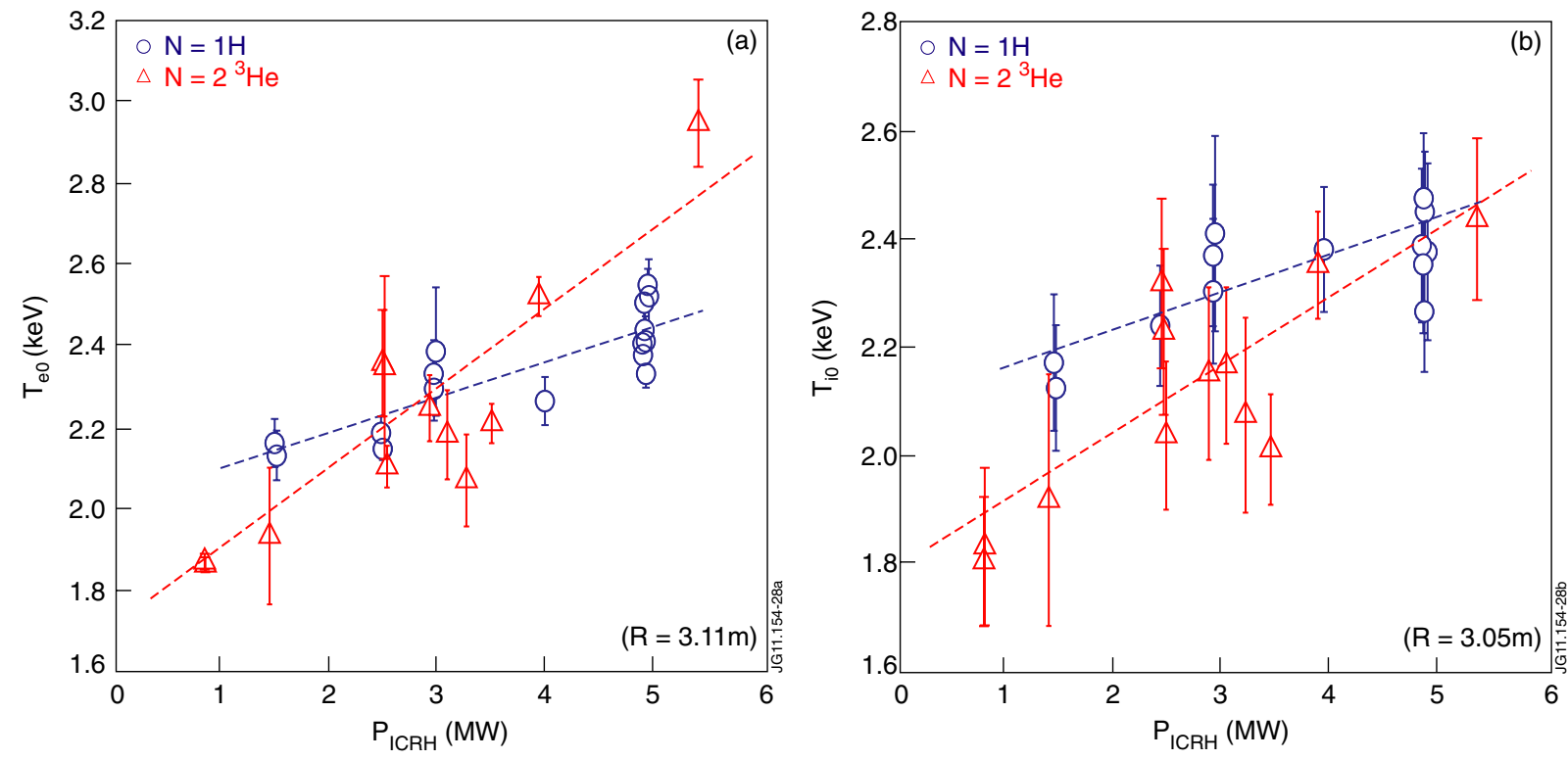

Figure 28. Central electron $(a)$ and ion $(b)$ temperatures plotted as a function of the applied ICRF power for the $N=1 \mathrm{H}$ (circles) and for the $N=2{ }^{3} \mathrm{He}$ (triangles) heating experiments.

that the central temperatures achieved in the $N=1 \mathrm{H}$ heating experiments (where core transport is prevailing) are smaller than those reached in the $N=2{ }^{3} \mathrm{He}$ heating discharges, as already suggested in sections 2.4 and 3.4.

A direct comparison of the overall heating performances of the two scenarios is depicted in figure 28, where the averaged electron $(a)$ and ion $(b)$ temperatures achieved in the $N=1$ $\mathrm{H}$ (circles) and $N=2{ }^{3} \mathrm{He}$ (triangles) heating experiments plotted as a function of the applied ICRF power are compared. The plasma densities were similar in all the time intervals and the slopes indicated in the two plots are the same as those discussed before in figures 11 and 24 .
One indeed sees that because the (core) transport losses increase faster with the applied RF power in the $N=$ $1 \mathrm{H}$ experiments, the increase in the central temperatures with ICRF power is slower than that observed in the ${ }^{3} \mathrm{He}$ experiments, where (edge) radiation losses are dominant. Furthermore, at a high RF power, the central electron temperatures reached in the $N=1 \mathrm{H}$ experiments are smaller than those achieved in the $N=2{ }^{3} \mathrm{He}$ heating pulses with the same applied power, but the $T_{\mathrm{i}}$ values are similar. It is interesting to note that for low power levels, both the electron and ion temperatures achieved in the $N=1 \mathrm{H}$ experiments are larger than those obtained in the $N=2{ }^{3} \mathrm{He}$ discharges. This 
is probably related to the higher resistivity (higher $Z_{\text {eff }}$ ) of the ${ }^{3} \mathrm{He}$ plasmas even before the application of the ICRF power, which leads to somewhat smaller equilibrium temperatures for the same input power and equilibrium parameters.

\section{Summary and discussion}

While the ICRF scenarios for the full-field DT phase of ITER have already been extensively explored in the past (see [30-34] and references therein), the heating schemes for the non-active half-field phase in hydrogen plasmas were not yet investigated carefully in large tokamaks. Recently, JET experiments have been designed to test some of these scenarios and numerical efforts for assessing their performance under ITER conditions have been put forward [35-37].

The results of the fundamental $\mathrm{H}$ majority heating $\left(B_{0}=\right.$ $2.65 \mathrm{~T}, f=42 \mathrm{MHz})$ and of the second harmonic $(N=2)$ ${ }^{3} \mathrm{He}$ ICRF heating $\left(B_{0}=2.65 \mathrm{~T}, f=51 \mathrm{MHz}\right)$ experiments recently performed in JET were presented. Although the RF parameters and the central plasma density foreseen for ITER's initial operation phase could be closely reproduced, the temperature was below that expected in ITER. As expected from theory, the experiments have shown that the two heating scenarios studied feature low ICRF power absorptivity leading to poor heating performance and enhanced plasma-wall interaction.

ICRF heating efficiencies in the range $\eta=P_{\mathrm{abs}} / P_{\mathrm{RF}}=$ $0.3-0.4$ were found for the fundamental $\mathrm{H}$ heating scheme and evidence of increased efficiency at larger plasma temperatures was observed. In addition, there were indications that the heating performance is improved when small amounts of ${ }^{4} \mathrm{He}$ are present in the hydrogen plasma. The acceleration of the ions was small: maximum $\mathrm{H}$ ion energies of approximately $30 \mathrm{keV}$ and $200 \mathrm{keV}$ were registered, respectively, with the horizontal and with the vertical NPA diagnostics when $5 \mathrm{MW}$ of ICRF power was applied. No indication of parasitic D/4 $\mathrm{He}$ absorption was found by any diagnostic, independently of the level of D NBI power applied. As a result of the low heating efficiency, relatively high radiation losses and poor plasma confinement, the equilibrium temperatures achieved after the application of ICRF power were modest.

For the $N=2{ }^{3} \mathrm{He}$ heating scenario, the heating efficiency varied from $\eta=0.1$ (at low ${ }^{3} \mathrm{He}$ concentrations) up to $\eta=0.4$ when ${ }^{3} \mathrm{He}$ concentrations above $20 \%$ were reached. Based on the central temperature responses to RF power modulation, it was seen that the increase in the heating efficiency with $X\left[{ }^{3} \mathrm{He}\right]$ is mainly due to enhanced ${ }^{3} \mathrm{He}$ ion cyclotron absorption rather than due to electron heating. While at low ${ }^{3} \mathrm{He}$ concentrations the discharges are fully dominated by electron heating, for $X\left[{ }^{3} \mathrm{He}\right]>20 \%$ the ion absorption overcomes the electron one. The heating efficiency did not show any clear trend with the plasma temperature in this case. Fast ${ }^{3} \mathrm{He}$ ions up to $160 \mathrm{keV}$ and $260 \mathrm{keV}$ were measured with the horizontal and vertical NPA diagnostics, respectively, when the ${ }^{3} \mathrm{He}$ concentration was about $8 \%$ and $5 \mathrm{MW}$ of RF power was applied. At higher concentrations, the RF-induced tails were even more modest suggesting that the ${ }^{3} \mathrm{He}$ dilution is more important than the increase in the heating efficiency for the formation of fast ion tails in these experiments. Unlike for the $N=1 \mathrm{H}$ majority heating case, a clear signature of parasitic beam ion acceleration was confirmed in the $N=2{ }^{3} \mathrm{He}$ heating experiments, in particular when highenergy PINIs were used. Again, the low heating efficiency associated with high radiation losses led to poor ICRF heating performance.

When comparing the overall performance of the two heating schemes, it was realized that while in the $N=2{ }^{3} \mathrm{He}$ scenario the radiation losses consist roughly half of the total losses, the transport losses dominate the fundamental H heating discharges (the radiation losses are about 25\%). This could explain the somewhat higher central temperatures reached in the former when similar RF power levels were applied, despite the similar heating efficiencies.

The numerical modelling of these scenarios [35] agreed well with the experimental results obtained but demonstrated that, unlike for good single-pass absorption cases, the modelling of weak absorption scenarios is very sensitive to the choice of the plasma parameters, in particular to the density and temperature profiles and to the main toroidal modes considered. The predictions of their performance in ITER [35-37], based on 1D and 2D full-wave modelling, show that these heating schemes will also suffer from poor single-pass absorption $(\eta<0.5)$ and that in the two cases electrons rather than ions will be primarily heated. In line with the experimental observations, the simulations have confirmed that increasing the plasma temperature is beneficial for $\mathrm{H}$ heating in the fundamental $\mathrm{H}$ scenario but temperatures as high as $25 \mathrm{keV}$ would be necessary to achieve similar ion and electron absorption under ITER conditions. For the $N=2$ ${ }^{3} \mathrm{He}$ heating case the simulations also confirm that a large amount of ${ }^{3} \mathrm{He}$ would be needed to achieve dominant ion heating. For the ITER baseline scenario $\left(X\left[{ }^{3} \mathrm{He}\right]=4 \%\right)$, $80 \%$ of the power is absorbed by the electrons via fast wave LD/TTMP and the single-pass absorptivity is only about $\eta=0.25$.

The results reported here and the respective numerical predictions for ITER suggest that the hydrogen phase of ITER will, in general, suffer from weak ICRF power absorption. This does not mean that it cannot be used to test the ICRF system at moderate power, since the coupling properties of the antenna are predominantly dependent on the scrape-off layer density and temperature profiles rather than on the ICRF absorptivity itself. However, the ICRF power level may be limited due to the high radiation levels inherent in weak absorption scenarios. A much better performance of the ICRF system is expected in ITER's half-field ${ }^{4} \mathrm{He}$ plasma phase, where $\mathrm{H}$ minority heating will be used and the heating efficiency is supposed to be large as long as the minority concentration remains modest [36]. However, other factors such as the use of $\mathrm{H}$ pellet injection for ELM pacing may contribute to increase the $\mathrm{H}$ concentration in these plasmas and it is very important to assess this effect on the ICRF heating efficiency both numerically and experimentally.

\section{Acknowledgments}

This work was supported by EURATOM and carried out within the framework of the European Fusion Development 
Agreement. The views and opinions expressed herein do not necessarily reflect those of the European Commission.

Euratom (C) 2012.

\section{References}

[1] ITER Team et al 1999 ITER Physics Basis Nucl. Fusion 392137

[2] Loarte A and Lamalle P 2010 private communication

[3] Lamalle P et al 2009 18th Topical Conf. on RF Power in Plasmas (Ghent, Belgium 2009) AIP Conf. Proc. 1187265

[4] Lerche E et al 2010 37th EPS Conf. on Plasma Physics (Dublin, Ireland, 2010) vol 34A (ECA) O4.121

[5] Kaye A et al 1994 Fusion Eng. Des. 24 1-21

[6] Challis C et al 1995 Fusion Eng. Des. 26 17-28

[7] Van Eester D et al 2009 Plasma Phys. Control. Fusion 51044007

[8] de la Luna E et al 2004 Rev. Sci. Instrum. 753831

[9] von Hellermann M G et al 1990 Rev. Sci. Instrum. 613479

[10] Korotkov A et al 1997 Nucl. Fusion 3735

[11] Afanasyev V et al 2003 Rev. Sci. Instrum. 742338

[12] Kiptily V et al 2009 Nucl. Fusion 49065030

[13] Kiptily V et al 2002 Nucl. Fusion 42999

[14] Hjalmarsson A et al 2003 Rev. Sci. Instrum. 741750

[15] Hellesen C et al 2010 Rev. Sci. Instrum. 81 10D337

[16] McCormick K et al 2005 Fusion Eng. Des. 74679

[17] Morgan P et al 1995 Rev. Sci. Instrum. 66606

[18] Coffey I et al 2004 Rev. Sci. Instrum. 753737
[19] Lerche E et al 2008 Plasma Phys. Control. Fusion 50035003

[20] Book D L 1987 NRL Plasma Formulary (Washington, DC: Naval research Laboratories)

[21] Van Eester D and Lerche E 2011 Plasma Phys. Control. Fusion 53092001

[22] Perkins F 1997 Nucl. Fusion 17 1197-224

[23] Mayoral M-L et al 2006 Nucl. Fusion 46 S550-63

[24] Van Eester D et al 2012 Minority and mode conversion heating in $\left({ }^{3} \mathrm{He}\right)-\mathrm{H}$ JET plasmas Plasma Phys. Control. Fusion 54074009

[25] Karney C 1986 Comput. Phys. Rep. 4183

[26] Eriksson L-G et al 1998 Nucl. Fusion 38265

[27] Kiptily V et al 2012 Fast ions in mode conversion heating $\left({ }^{3} \mathrm{He}\right)-\mathrm{H}$ plasmas in JET Plasma Phys. Control. Fusion 54074010

[28] Czarnecka A et al 2012 Impurity production from the ICRH antennas in JET Plasma Phys. Control. Fusion 54074013

[29] Huber A et al 2007 Fusion Eng. Des. 821327

[30] Start D et al 1999 Nucl. Fusion 39321

[31] Strachan J et al 1997 Plasma Phys. Control. Fusion $39 \mathrm{~B} 103$

[32] Eriksson L-G et al 1999 Nucl. Fusion 39337

[33] Van Eester D et al 2002 Nucl. Fusion 42310

[34] Dumont R 2009 18th Topical Conf. on RF Power in Plasmas (Ghent, Belgium, 2009) AIP Conf. Proc. 118797

[35] Lerche E et al 2011 Plasma Phys. Control. Fusion 53124019

[36] Lerche E et al 2011 19th Topical Conf. on RF Power in Plasmas (Newport, RI)

[37] Budny R et al 2012 Benchmarking ICRF full-wave solvers for ITER Nucl. Fusion $\mathbf{5 2} 023023$ 